\title{
CHARACTERISTICS AND ANALYTICAL METHODS OF MANNITOL: AN UPDATE
}

\author{
ALANKAR SHRIVASTAVA ${ }^{1}$, SANJAY SHARMA ${ }^{2}$, MONIKA KAURAV ${ }^{1}$, ABHISHEK SHARMA ${ }^{1}$
}

${ }^{1}$ KIET Group of Institutions (KIET School of Pharmacy), ${ }^{2}$ NMIMS's School of Pharmacy and Technology Management, Quality Assurance Email: alankar.shrivastava@kiet.edu

Received: 16 May 2021, Revised and Accepted: 28 Jul 2021

\begin{abstract}
Mannitol is an organic compound, a widely distributed natural sugar alcohol in nature. It is found in various plant species and produced by many microorganisms. Mannitol is about $50 \%$ as sweet as sucrose, has a low glycaemic index and inert nature, making it suitable to be used in many food products. Mannitol is an osmotic diuretic administering through the iv route and having many clinical usages and is one of the well-known excipients in many different types of formulations. This is also used to increase the dissolution of drugs having solubility problems. Mannitol can be used as a drug and excipient. The goal of this work was to summarize the important physicochemical properties, mechanism of action, production, applications, incompatibilities, polymorphism, and particularly the analytical methodologies published in the last five decades for quantification. Relevant articles related to analytical methods were identified through a search of the English-language literature indexed in Medline, PubMed, ScienceDirect and google scholar from 1970 to till date. The search terms were benign estimation of mannitol, determination of mannitol, methods for determination of mannitol, HPLC and Spectrophotometry method for estimation of mannitol. The methods described in USP, IP, and BP are also described. The presented review also outlines the further scope of research in the field of development of analytical methods.
\end{abstract}

Keywords: Analytical methods, Mechanism of action of mannitol, Mannitol, Biosynthesis, Polymorphism

(C) 2021 The Authors. Published by Innovare Academic Sciences Pvt Ltd. This is an open-access article under the CC BY license (https://creativecommons.org/licenses/by/4.0/) DOI: https://dx.doi.org/10.22159/ijap.2021v13i5.42068. Journal homepage: https://innovareacademics.in/journals/index.php/ijap

\section{INTRODUCTION}

One of the naturally occurring alcohols found in vegetables and fruits is Mannitol, and it is widely used as an osmotic diuretic [1]. The Joseph Louis Proust discovered mannitol in the year 1806 [2].
This is sweet and available as a white, odorless crystalline powder or free-flowing granules [3]. The $D$-enantiomer of Mannitol, $D$-Mannitol ((2R, 3R, 4R, 5R)-hexane-1,2,3,4,5,6-hexol) (fig. 1) can be used by IV or oral route [4]. The physical properties of Mannitol are given under table 1.

Table 1: Physical properties of mannitol

\begin{tabular}{lll}
\hline Properties & Remark & Reference \\
\hline CAS No. & $69-65-8$ & [1] \\
Synonyms & $D-M a n n i t o l$, mannite, manna sugar, cordycepic acid, manicol, mannidex, osmitrol, osmosal, resectisol \\
Mol formula & $\mathrm{C}_{6} \mathrm{H}_{14} \mathrm{O}_{6}$ \\
Mol. weight & 182.17 \\
Melting Point & $166-168{ }^{\circ} \mathrm{C}$ \\
Boiling Point & $290-295{ }^{\circ} \mathrm{C}$ \\
pKa at $18^{\circ} \mathrm{C}$ & 13.50 & 1 gm in $\sim 5.5$ gm water, 83 ml alcohol, more soluble water (hot), Insoluble-ether, Soluble-pyridine, \\
Solubility (water) & aniline, aqueous solutions of alkalies, 1 gm dissolves in 18 ml glycerol \\
& Media & Solubility \\
Solubility (others) & Ether & Insoluble \\
& Pyridine & Soluble \\
& Aniline & Soluble \\
& Alkalies (Aq.) & Soluble \\
& Glycerol & 1 gm in 18 ml \\
\hline
\end{tabular}

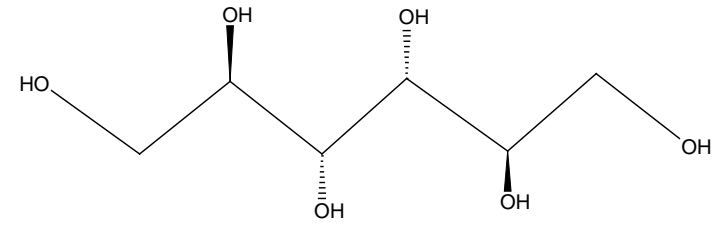

Fig. 1: Structure of mannitol

Some clinical conditions, e. g., acute renal failure and cerebral edema, IV administration of Mannitol induces dieresis [5]. Mannitol is also used in neurology and neurosurgery because of its property of causing dehydration [6]. Mannitol is recommended in increased ocular pressure [7]. This is also used for the assessment of renal function since it is excreted unchanged through the kidneys, thus accumulated in abnormal renal function resulting in cellular dehydration [8]. Mannitol is also known to cause temporary disruption of the blood-brain barrier allowing better penetration of chemotherapeutic drugs $[9,10]$. Mannitol is the most used enteric contrast in magnetic resonance enterography due to better depiction, more availability, and lower cost [11]. Mannitol is clinically proved in improving airway function in cystic fibrosis with an acceptable safety profile [12]. The IDPM bronchial provocation test to evaluate bronchial responsiveness in asthma [13].

The market size of mannitol is USD 209.4 million [14] and possibly reaching USD 418.3 million by 2024 [15]. Mannitol is one of the essential medicines in the list of most of countries [16]. Due to its sweet taste (about half than sucrose), low hygroscopicity and cooling sensation (heat of solution is $-28.9 \mathrm{cal} / \mathrm{g}$ at $25^{\circ} \mathrm{C}$ ), it is also used in the preparation of chewable tablets [17]. Mannitol is one of the low-digestible sugar with negligible or minimum effect on blood 
glucose levels and low calories (1.6 cal/g) is additional advantage, especially for diabetic people [18].

Mannitol is quite common excipient diluent $(10-90 \%, \mathrm{w} / \mathrm{w})$ in tablet formulations [19]. It is soluble in water, non-hygroscopic, non-toxic, and has moderate resistance towards heat [20]. This is generally recognized as safe and frequently used in solid dosage forms [21]. High porous particles increase the surface area, which is an additional advantage due to which, when incorporated with API, water solubility and dissolution rate increases [22]. Other than this, Mannitol is also used for thickening aqueous antacid suspensions of $\mathrm{Al}(\mathrm{OH})_{2} \quad(>7 \%)$ and as one of the components of SR tablet formulations and plasticizers in soft-gelatin capsules [23]. Mannitol is also a free-radical scavenger, which may be the reason for acting as a neuroprotectant [24]. Mannitol hexanitrate is used in the treatment of hypertension because of its vasodilatory action [25].

Mannitol is slightly acidic ( $\mathrm{pH}$ 6.3); thus it requires additional alkaline components in proprietary preparations for adjustment of $\mathrm{pH}$ and usually, sodium carbonate is used [26]. The solutions of mannitol $(>10 \% \mathrm{w} / \mathrm{v})$, if kept at room temperature, show the appearance of crystals but can be solubilized by increasing the temperature [27]. For example, the solubility of mannitol at 14 and $25^{\circ} \mathrm{C}$ is $13 \%$ and $18 \% \mathrm{w} / \mathrm{v}[28,29]$.

Mannitol increases the shelflife of foodstuffs by reducing the tendency of sugars to crystallize [29]. Mannitol and sorbitol are isomers; the only difference is the orientation of the C2 hydroxyl group [30]. The solution form of mannitol is not affected by dilute acids or alkalis, or oxidation by oxygen present in the atmosphere provided catalysts are not present to initiate the reaction [31]. Mannitol does not undergo Maillard browning and caramelization [32] because of not possessing a carbonyl group in its structure [33]. The literature review related to this communication is presented under fig. 2. Relevant articles related to analytical methods were identified through a search of the Englishlanguage literature indexed in Medline, PubMed, ScienceDirect and google scholar from 1970 to till date. The search terms were benign estimation of mannitol, determination of mannitol, methods for determination of mannitol, HPLC and Spectrophotometry method for estimation of mannitol.

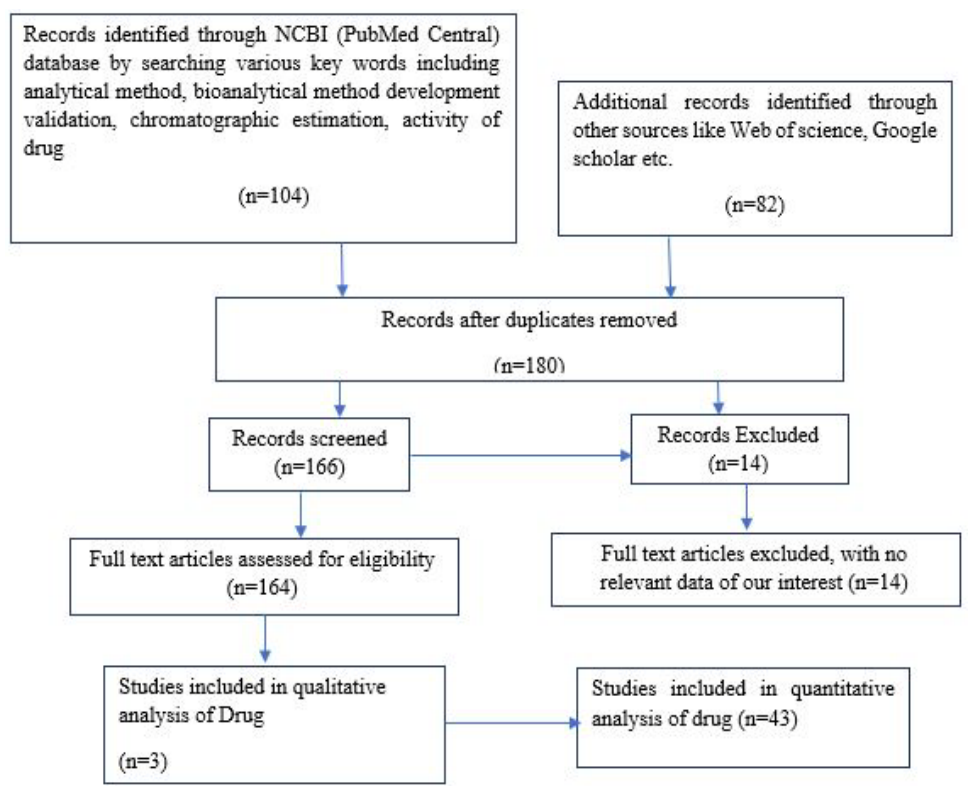

Fig. 2: Data of literature review of mannitol (1970-till date)

\section{Pharmacokinetics and pharmacodynamics}

Mannitol, when administered orally, is absorbed poorly by the GI tract and may cause osmotic diarrhea. It should be given parenterally for systemic effect and excreted by kidneys within 30$60 \mathrm{~min}$. Mannitol is not reabsorbed by tubular reabsorption and secretion [34]. About $80 \%$ of the given dose is excreted unchanged in the urine in $3 \mathrm{~h} \mathrm{[34]}$.

After being administered intravenously, it causes a reduction of intracranial pressure by increasing the tonicity of blood plasma. The blood-brain barrier restricts the entry of mannitol, but increased tonicity draws water from the parenchyma of the brain reducing intracranial pressure. This water is then excreted through the kidney in urine and thus mannitol indirectly increases the mild dehydration of brain cells [35]. The tonicity of blood plasma increases by mannitol and draws water into intravascular space from out vitreous humor in the eye causing lowering of intraocular pressure and damage to the retina is prevented [36]. Mannitol along with fluid increased the urine output in fluid overload caused by oliguria. But the usage of mannitol in such cases becomes obsolete since it may precipitate acute tubular necrosis [37]. During excretion it draws water and other toxic materials too, decreasing their concentration in blood [38]. IDH is one of the common symptoms due to a decrease in osmolarity of blood serum. However, the benefit of mannitol in long term for the prevention of IDH is not much clear $[39,40]$.

\section{Mannitol production methods}

There are many methods through which mannitol is either extracted naturally (Plants) or produced via different chemical or microbial and biosynthesis methods. For mannitol extraction from plants sources, supercritical fluid and subcritical fluid technology was extensively employed $[41,42]$. In the chemical synthesis method, mannitol production is induced in the presence of catalyst by the hydrogenation of $D$-glucose and $D$-fructose mixtures [43-45]. In the enzymatic method, the conversion of D-fructose directly into Dmannitol allowed in the vicinity of $\mathrm{MDH}$ and FDH allowed in presence of cofactors (NADH or NADPH) (fig. 4A, 4B and 4C) [46, 47]. In the fermentation method production of mannitol is done via reduction of different substrates such as fructose, glucose, glycerol, and sucrose via different microorganism strains such as Aspergillus candidus, Lactobacillus intermedius, Candida Parapsilosis, Penicillium (fig. 4) [48-53]. Further to increase mannitol production, engineered strain prepared by knocked out technology such as Lactobacillus lactis, Leuconostoc pseudomesenteroides. S. cerevisiae etc [54-58]. Currently, recombinant strain of Escherichia coli with insertion of other strain genes encoded to increase or facilitate production most commonly employed (fig. 4) [59-61]. 

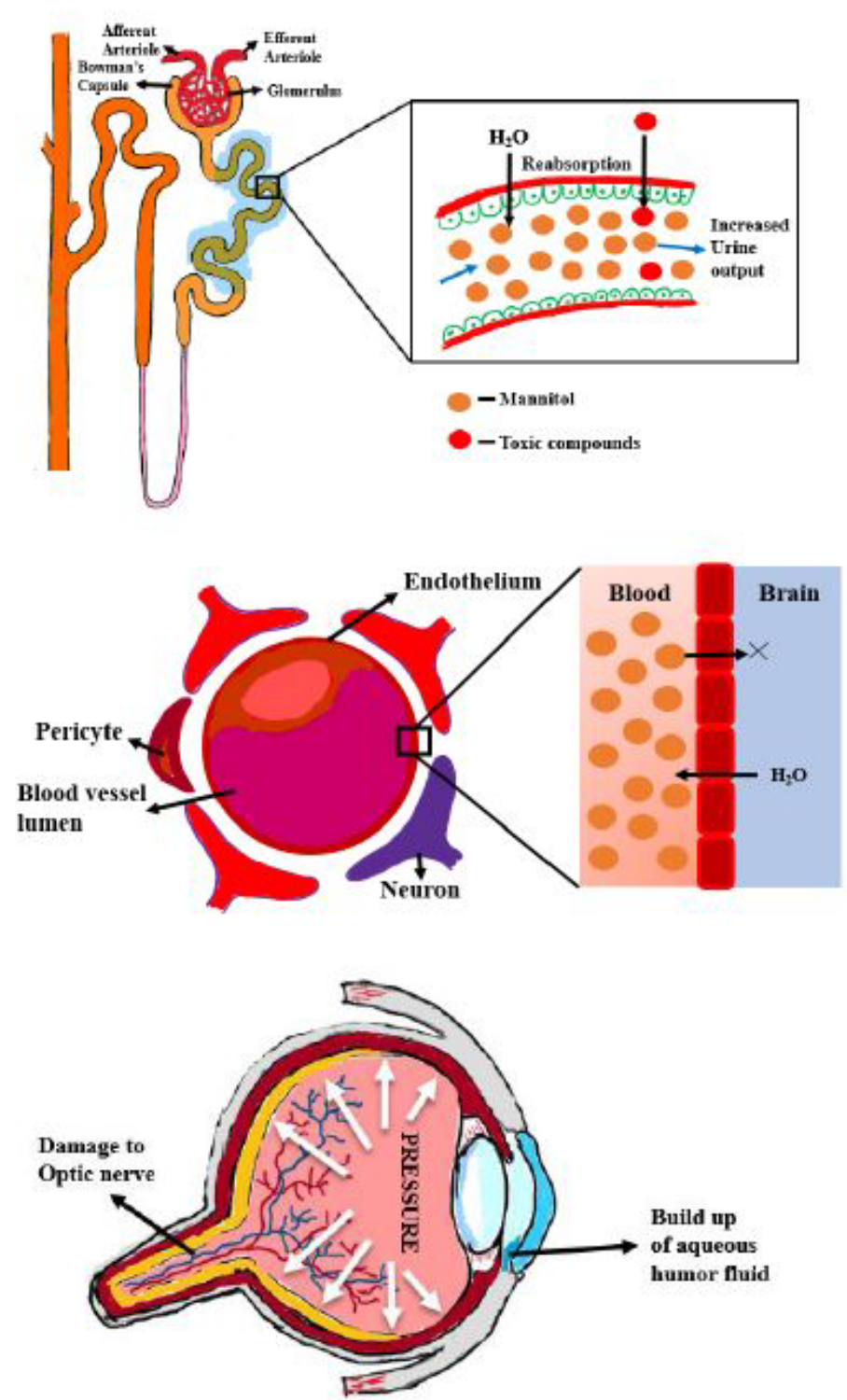

Fig. 3: Mechanism of action of mannitol (a) Proximal convoluted tubules, (b) Blood-brain barrier, and (c) Intraocular pressure (IOP)

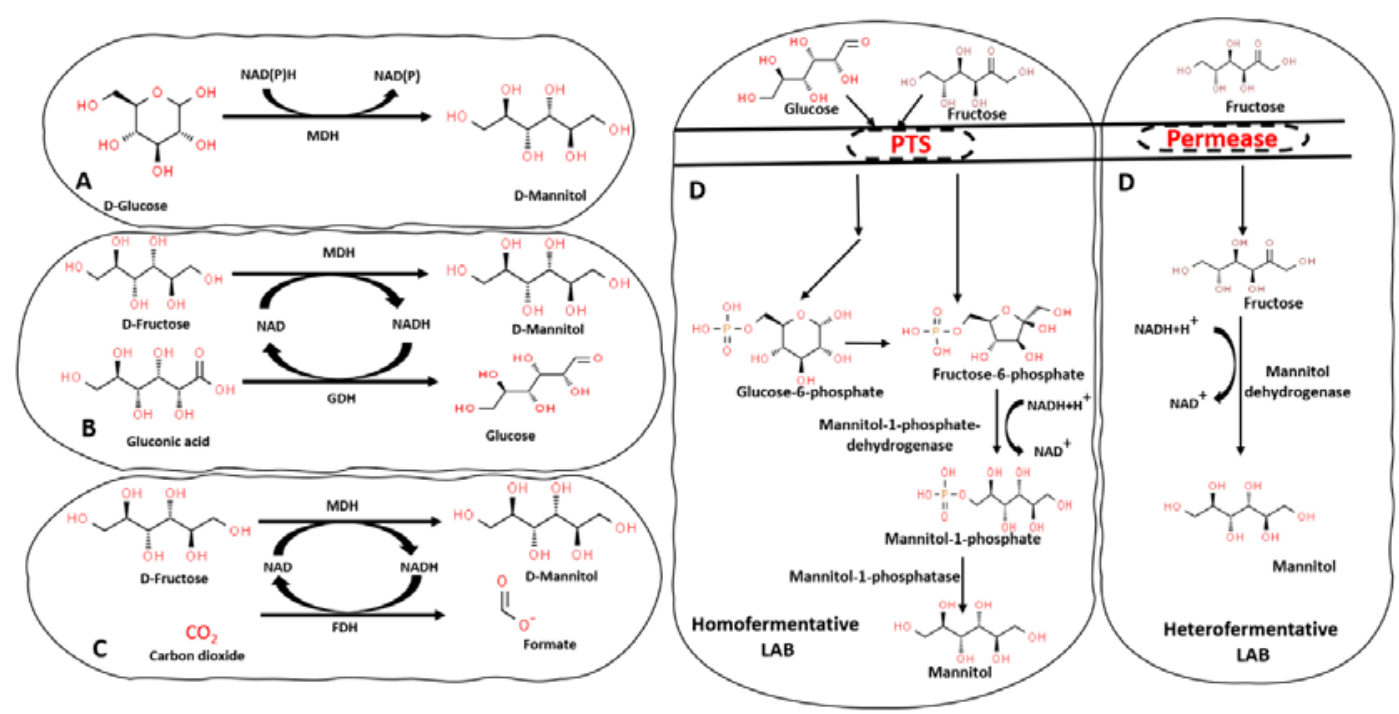

Fig. 4: Production of mannitol 


\section{Applications}

Mannitol is considered and listed as a GRAS sweetener according to FDA, thus widely used as a food additive in the food industry to mask the bitter taste of ingredients. Its sweetness is about $50-70 \%$ that of sucrose with cooling taste due to negative heat of solution. In humans, $75 \%$ of the total consumed mannitol's fermentation occurs in the intestinal flora and only $25 \%$ is absorbed before urinary excretion. Previous works of literature also suggest that mannitol is prebiotic via altering large intestinal fermentation mechanism and produces more butyrate and propionate amounts [62]. After absorption mannitol, interacts with $\mathrm{NAD}^{+}$and produces fructose and acetyl CoA. In the presence of fructokinase, fructose is converted into the two forms of fructose diphosphates that arrive further at the triose-phosphate cycle for the metabolism of mannitol. So, the overall process is insulin-independent; thus no significant alteration in blood glucose level occurs. In this respect, mannitol can also be used in food products for diabetic people. Mannitol via reducing the crystallization tendency of carbohydrates will also be able to increase the shelf-life of food products [43]. Due to the low hygroscopic nature of crystalline mannitol in the highly humid environment, too, it provides stability to products as additives [49]. However, if daily mannitol intake exceeds above $20 \mathrm{~g}$, it may exert a laxative effect [48].

As already mentioned, that mannitol has low hygroscopic nature, produces negative heat of solution, masks the unpleasant bitter taste of ingredients, and provides sweet, cooling taste, thus most commonly used excipient in granular powders, chewable tablets, and gums $[63,64]$.

In injectable formulations, mannitol can be employed as a dehydrating agent and as a diuretic in the treatment of glaucoma and hydrocephalic condition. In freeze-dried reconstitution type injection, the addition of mannitol is done as suspending and thickening agent and it also provides antioxidant effects [65]. Hypertonic mannitol solution enhances the permeation of water- soluble drugs and macromolecules in CNS chemotherapy via surging permeability of the blood-brain barrier [66].

Mannitol also works as a strong antioxidant, thus scavenge free hydroxyl radical to reduce the damage against neurological disorders. The free radical activity of particles $<10 \mu \mathrm{m}$ in air pollution, mannitol may reverse the depletion of supercoiled plasmid DNA [67]. In few previous works, Eggleston et al. and Eggleston and Harper (2006) found that mannitol can be used as a sensitive indicator via the enzymatic method (mannitol dehydrogenase catalyst) to determine mannitol deterioration in sugarcane industries $[68,69]$.

\section{Polymorphism and incompatibility studies}

Mannitol shows multiple polymorphic phases, i.e., $\alpha, \beta, \delta[70,71]$, and all are approved for as carriers of dry powder inhalers [20]. The $\alpha$ form shows the fastest growth rate and its seeds tend to form the same type of polymorph. Whereas, seeds of $\alpha$ or $\beta$ form appears from $\beta$ polymorph and $\alpha$ form forms $\delta$ polymorph [72-74]. Although the $\delta$ form is the least stable, its superior compaction properties increasingly increase its importance as an excipient, particularly for tablet manufacturing. The most thermodynamically stable polymorph is the $\beta$-form. The $\delta$ form, however, showing the least stability but stable even up to $5 \mathrm{y}$ up to $25{ }^{\circ} \mathrm{C}$ under dry conditions $[22,74]$. Mannitol shows five different crystal planes; (011), (010), (120), (110) and (210) [75]. The $\delta$ form crystals are available commercially and can be used for the preparation of two other polymorphic forms. The $\alpha$ polymorph may be prepared by antisolvent precipitation using acetone and water. The melt crystallization method can be used for the preparation of $\beta$ form [76].

Cares-Pacheco et al., [77] described $\alpha$ polymorph was found to be the most energetically active form supporting the usage of this form in a dry powder inhaler (DPI), due to its needle-shaped crystalline structure. The Raman spectra of $\alpha$ and $\beta$ forms are quite similar; only the $\delta$ polymorph shows unique peaks helpful in distinguishing from other polymorphic forms. Some of the differences are described in table 2 and structural differences are shown in fig. 5 .

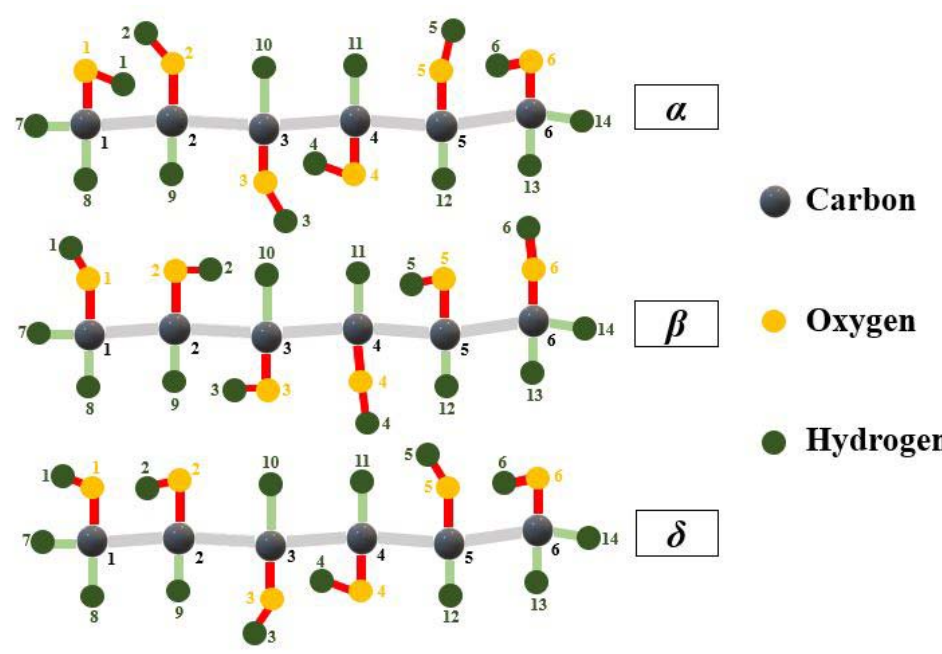

Fig. 5: The structures of polymorphs of mannitol $(\alpha, \beta$, and $\delta)$

Table 2: Some differences of properties of polymorphs of mannitol [78, 79]

\begin{tabular}{llll}
\hline Properties & $\boldsymbol{\alpha}$ & $\boldsymbol{\beta}$ & $\boldsymbol{\delta}$ \\
\hline Stability & Intermediate stability & Most stable & Least stable \\
Types & orthorhombic & orthorhombic & $52.1 \pm 0.9$ \\
Enthalpy of fusion $\left(\mathrm{kJ} \mathrm{mol}^{-1}\right)$ & $52.1 \pm 0.9$ & $53.5 \pm 0.4$ & 1054 and $1250 \mathrm{~cm}^{-1}$ \\
Raman spectra (single peak) & $1030,1130,1355 \mathrm{~cm}^{-1}$ & 876,1037 and $1136 \mathrm{~cm}^{-1}$ & Lath, colorless \\
Shape & Needle fragment, colorless & Lath, colorless & $165.8^{\circ} \mathrm{C}$ \\
\hline Melting point & $166^{\circ} \mathrm{C}$ & $166.5^{\circ} \mathrm{C}$ & \\
\hline
\end{tabular}

Mannitol is neutral towards atmospheric oxygen in catalysts' absence and stable in the dry state [80]. Maillard reaction is a coloring reaction between reducing sugars and amino groups [81] to form Schiff bases, but in the absence of any carbonyl group, mannitol is stable even at the high 
temperature and does not generate different flavor and brown color [82]. As a rule, drug excipients are a significant part of a medication item, with the dynamic compound present in generally modest quantities [83]. The incompatibilities are given in table 3 .

Table 3: Mannitol incompatibilities with drugs

\begin{tabular}{|c|c|c|}
\hline Drug & Remark/Possible mechanism & References \\
\hline Atenolol & Hydrogen-bond between the- $\mathrm{NH}_{2}$ of atenolol and mannitol's- $\mathrm{OH} / \mathrm{C}-\mathrm{H} / \mathrm{CH}_{2}$ groups & {$[84,85]$} \\
\hline Acetaminophen & DSC peaks shows interaction with mannitol & [86] \\
\hline Cimetidine & Transit time in small intestine shortened after administering mannitol tablet and solution & [87] \\
\hline Granisetron & FTIR and DSC studies shows interaction & [88] \\
\hline Carbamazepine & DSC studies shows incompatibility with mannitol & [89] \\
\hline Omeprazole & Variations in ratios of $S$-and $R$-omeprazole sodium & [90] \\
\hline Sildelafil & API curve changes in DSC possibly due to degradation & [91] \\
\hline Primaquine & FTIR indicates intermolecular links and possible chemical incompatibility & [92] \\
\hline
\end{tabular}

\section{Analytical methods}

The analytical method should be acceptable for its intended purpose [93]. The scientific basis for the development of formulation is done through information gained during pharmaceutical development. Diluents or excipients are added to form appropriately sized solid dosage forms (e. g., tablets) and constitute a major proportion. They may also be used as a coating material for the protection of undesirable organoleptic properties of drug substances [94]. To obtain data related to quantitation, analytical methods are required. For this purpose, various types of instrumental methods are used for the determination of analytes, e. g., chromatography methods, spectrophotometry methods, electroanalytical methods, and hyphenated techniques are widely used. The available literature also indicates that Mannitol can be determined by using a few of these versatile methods.

The UV spectrophotometry, IR, and $\mathrm{H}^{1}$ NMR graphs of mannitol are shown under fig. 6,7 , and 8 , respectively.

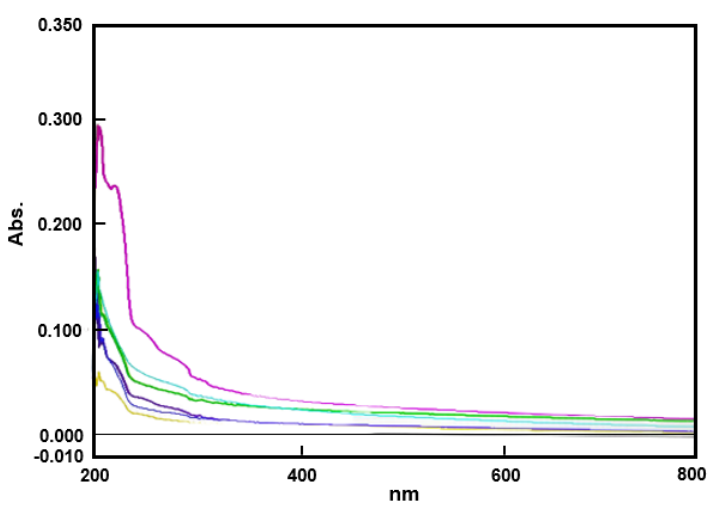

Fig. 6: Overlay UV spectra of mannitol $(1,5,10,20,50,100 \mu \mathrm{g} / \mathrm{ml}$

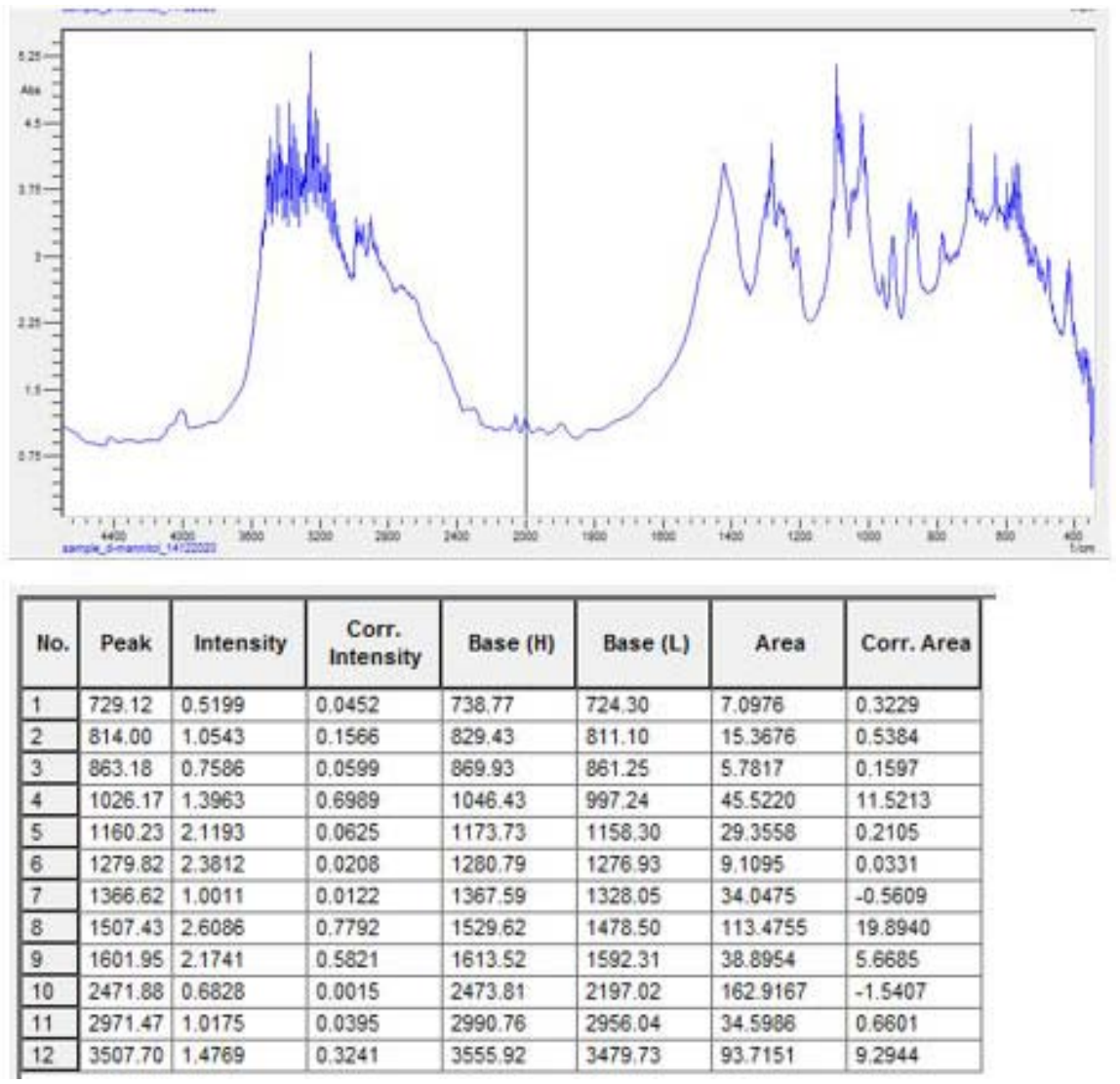

Fig. 7: IR spectra of mannitol 


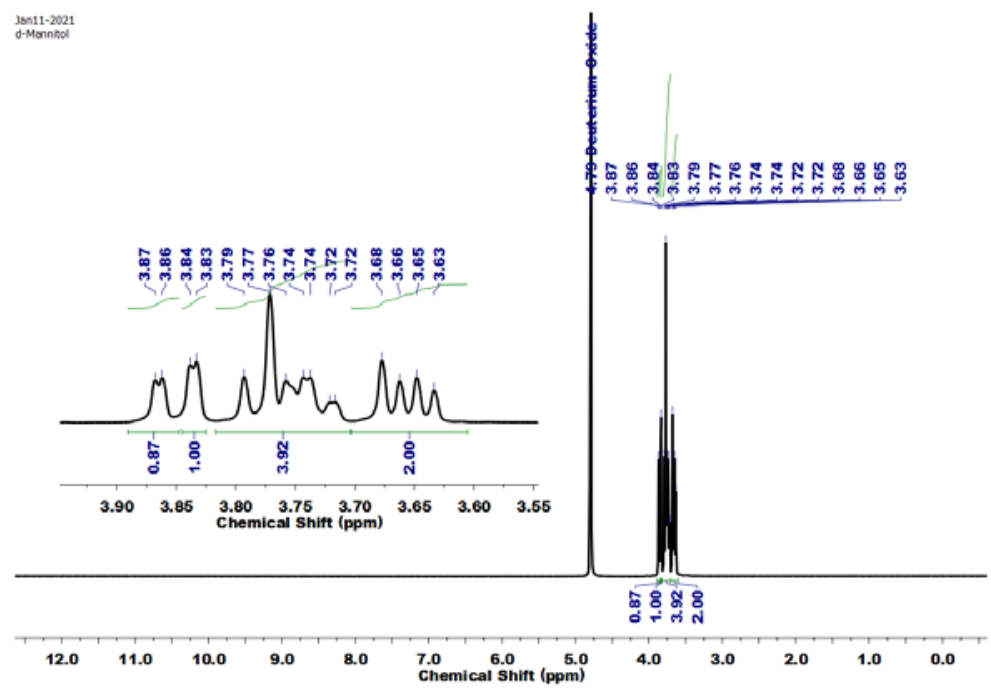

Fig. 8: H-NMR of mannitol

\section{Spectrophotometry methods}

Spectrophotometry is a widely accepted analytical technique in the pharmaceutical industry for different analytical processes. The applications of spectrophotometry are expanding all the time [95].

Mannitol was also determined by using an enzyme for successful estimation by UV spectrophotometry. For this, mannitol dehydrogenase was used by many researchers. A few microorganisms like Pseudomonas fluorescense and Rhodobacter sphaeroides, Thermotoga maritima, and Thermotoga neapolitana are known to produce mannitol dehydrogenase. These enzymes convert mannitol into fructose in the presence of Nicotinamide adenine dinucleotide (NAD+), an essential cofactor that participates in fundamental biological processes. The reduced form of $\mathrm{NAD}+$ absorbs light in the UV region and can be measured at $340 \mathrm{~nm}$
[96]. The absence of chromophore in the chemical structure is a possible reason for the absence of direct spectrophotometry analysis. This is also evident by scanning dilutions in the UV visible range (fig. 6).

The spectrophotometry methods published in various sources are presented in table 4 .

\section{Chromatography methods}

The complex samples require separation of analyte first before final analysis and this is well evident that chromatography methods are the most suitable methods for the same. Currently, many different chromatography methods are available, classified mainly based on the mobile phase [105].

The published chromatography methods are given in table 5 .
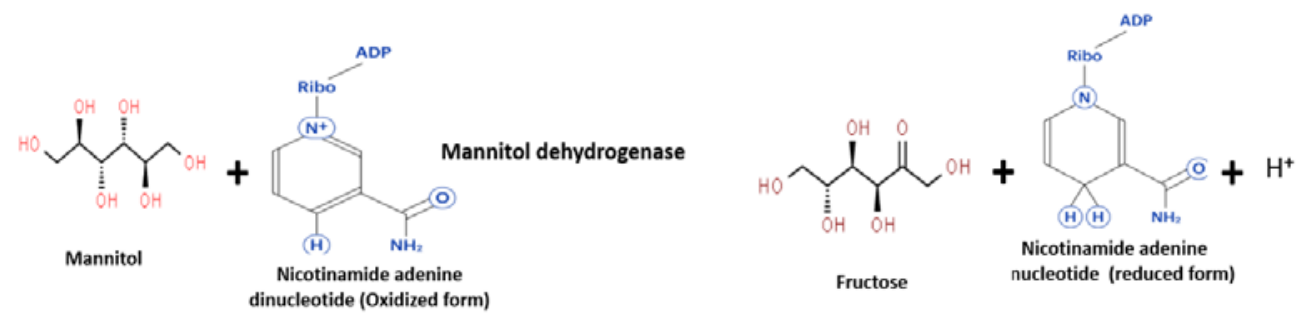

Fig. 9: Reaction of mannitol with NAD+in the presence of mannitol dehydrogenase

Table 4: Summary of spectrophotometry methods of mannitol

\begin{tabular}{llll}
\hline Method & Source of enzyme & wavelength & Application \\
\hline UV & $\begin{array}{l}\text { Lactobacillus brevis } \\
\text { Lactobacillus } \\
\text { brevis or Leuconostoc mesentroids } \\
\text { Agaricus bisporus }\end{array}$ & $340 \mathrm{~nm}$ & Serum samples (dogs) \\
DV & $340 \mathrm{~nm}$ & [97] \\
[98] & $340 \mathrm{~nm}$ & $\begin{array}{l}\text { Determination in urine } \\
\text { polysaccharides and glycoproteins }\end{array}$ \\
Fluorometric & - & $\lambda \mathrm{ex}=350 \mathrm{~nm}$ and $\lambda \mathrm{em}=460$ & Fungal and mycorrhizal extracts \\
assay & & $\mathrm{nm}$ & \\
[99] & - & $\lambda_{\mathrm{ex}}=340 \mathrm{~nm}$ and $\lambda_{\mathrm{em}}=455 \mathrm{~nm}$ & \\
UV & Leuconostoc mesenteroides & $340 \mathrm{~nm}$ & human blood or serum \\
UV & Leuconostoc mesenteroides & $340 \mathrm{~nm}$ & Determination in juice of sugarcane \\
UV & Mannitol dehydrogenase. & $340 \mathrm{~nm}$ & lactulose-mannitol ratio in urine \\
UV & Mannitol dehydrogenase & $340 \mathrm{~nm}$ & Urine \\
UV & Mannitol dehydrogenase & $340 \mathrm{~nm}$ & Urine \\
\hline
\end{tabular}


Table 5: Summary of chromatography methods for mannitol

\begin{tabular}{|c|c|c|c|c|c|}
\hline Method & Detector & Column & Conditions & Application & Reference \\
\hline GLC & FID & $\begin{array}{l}0.61 \mathrm{~m} \times 0.32 \mathrm{~cm} .(2 \mathrm{ft} . \times 0.125 \\
\text { in.) } \mathrm{OD} \text {, stainless steel column }\end{array}$ & $\begin{array}{l}\text { Column (isothermal), injector and } \\
\text { detector temperature } 175,175 \text {, and } \\
260{ }^{\circ} \mathrm{C}\end{array}$ & $\begin{array}{l}\text { Sorbitol and mannitol in } \\
\text { aqueous solutions }\end{array}$ & {$[106]$} \\
\hline GC & FID & $\begin{array}{l}\text { Glass columns (Borosil), } 1.22 \\
\mathrm{~m} \times 0.64 \mathrm{~cm} \mathrm{OD}\end{array}$ & $\begin{array}{l}\text { Isothermal, Temperature: detector, } \\
220^{\circ} \text {, injection port, } 240^{\circ} \text {. } \\
\text { His flow rate } 55 \mathrm{ml} / \mathrm{min} \text {, inlet } \\
\text { pressure } 40 \mathrm{psi} \text {. }\end{array}$ & $\begin{array}{l}\text { Sorbitol and Mannitol in } \\
\text { Pharmaceuticals }\end{array}$ & [107] \\
\hline $\mathrm{GC}$ & MS & $\begin{array}{l}\text { Glass columns }\left(6^{\prime \prime}, 1 / 3{ }^{\prime}\right) \text {, } \\
\text { packed with } 3 \% \text { OV-255 }\end{array}$ & $\begin{array}{l}\text { Temperature: Injection port, } 250^{\circ} \mathrm{C} \\
\text { and column, } 212^{\circ} \mathrm{C} \text {. }\end{array}$ & $\begin{array}{l}\text { Quantification } \\
\text { in CSF with six other sugars }\end{array}$ & [108] \\
\hline GC & FID & $\begin{array}{l}9 \mathrm{ft} \times 2 \mathrm{~mm} \text {, packed with } \\
\text { Silar } 10 \mathrm{C}, \text { Chromosorb W/AW } \\
(10 \%)\end{array}$ & $\begin{array}{l}\text { Temperature: Column, } 200{ }^{\circ} \mathrm{C} \text {; } \\
\text { injector, } 250{ }^{\circ} \mathrm{C} \text {; detector, } 330^{\circ} \mathrm{C} \text {; } \mathrm{N}_{2} \\
\text { flow } 20 \mathrm{ml} / \mathrm{min}\end{array}$ & $\begin{array}{l}\text { with two other sugars in } \\
\text { chewing gum and sorbitol in } \\
\text { mints }\end{array}$ & [109] \\
\hline HPLC & RI & $\begin{array}{l}\text { Sugar Pak I }(30 \mathrm{~cm} \times 6.5 \mathrm{~mm}) \\
\text { i.d., } 85^{\circ} \mathrm{C} \text {, pre-column filter }\end{array}$ & Mobile phase: $\mathrm{H}_{2} \mathrm{O}$, Flow: $0.4 \mathrm{ml} / \mathrm{min}$ & Analysis with four other sugars & {$[110]$} \\
\hline HPLC & RI & $\begin{array}{l}3 / 8 \mathrm{in} . \times 2 \mathrm{ft} \text { and } 10 \mu \mathrm{m} \text { (end } \\
\text { fittings) }\end{array}$ & $\begin{array}{l}\text { Mobile phase: Water-methanol } \\
(65+35) \text {, flow rate, } 2.0 \mathrm{ml} / \mathrm{min}(800 \\
\text { psig); } 55^{\circ} \mathrm{C}\end{array}$ & $\begin{array}{l}\text { In chewing gums and } \\
\text { confections with two other } \\
\text { sugars }\end{array}$ & {$[111]$} \\
\hline GLC & MS & $\begin{array}{l}\text { Glass column }(1 \mathrm{~m} \times 2-\mathrm{mm} \text { i.d. }) \\
\text { packing, } 0 \mathrm{~V}-17(3 \%)\end{array}$ & $\begin{array}{l}\text { Ionization curr, } 300 \mu \mathrm{A} \text {; Ionization } \\
\text { nrg, } 70 \mathrm{eV} \text {; accelerating volt, } 3.0 \mathrm{kV} \text {; } \\
\text { Temperatures: injector, } 280^{\circ} \mathrm{C} \text {, } \\
\text { column, } 270^{\circ} \mathrm{C} \text {, ion source } 250^{\circ} \mathrm{C} \text {. Ion } \\
\text { multiplier voltage: } 1.2-1.4 \mathrm{kV} \text {. }\end{array}$ & With sorbitol (in plasma) & [112] \\
\hline $\mathrm{GC}$ & MS & $\begin{array}{l}\text { Glass column, } 1.5 \mathrm{~m} \times 2.0 \mathrm{~mm} \\
\text { (i.d.), packing } 3 \% \mathrm{SP}-2340 \\
\text { (75:25, cyanopropyl: methyl } \\
\text { silicone gum, } 100 / 120 \text { mesh). }\end{array}$ & $\begin{array}{l}\text { Isothermal; } 70^{\circ} \mathrm{C} / 1 \mathrm{~min} \text {, then } 270^{\circ} \mathrm{C} \text {, } \\
12^{\circ} \mathrm{C} / \mathrm{min}\end{array}$ & $\begin{array}{l}\text { With } 7 \text { other carbohydrates in } \\
\text { serum }\end{array}$ & [113] \\
\hline HPLC & $\begin{array}{l}\text { Differenti } \\
\text { al RI }\end{array}$ & $\begin{array}{l}\text { Column.-300 } \mathrm{mm} \times 7.8 \mathrm{~mm}, 8 \% \\
\text { crosslinked cation exchange }\end{array}$ & Mobile phase: $\mathrm{H}_{2} \mathrm{O}$, flow: $1.0 \mathrm{ml} / \mathrm{min}$ & $\begin{array}{l}\text { With } 2 \text { other carbohydrates in } \\
\text { meat products }\end{array}$ & {$[114]$} \\
\hline GC & MS & $\begin{array}{l}\text { Glass columns }(1 \mathrm{~m} \times 4 \mathrm{~mm} \\
\text { I.D.) packing, } 3 \% \text { Dexsil- } 400 \text { or } \\
\text { with } 1: 1,3 \% \text { OV-l/OV- } 17 \text {. } \\
\text { temperature, } 210{ }^{\circ} \mathrm{C}\end{array}$ & $\begin{array}{l}\text { Injection port } 20 \mathrm{kPa} \text { (Pressure), flow } \\
1.1 \mathrm{ml} / \mathrm{min} \text {. Split ratio } 10: 1 . \mathrm{EI} 70 \mathrm{eV}\end{array}$ & $\begin{array}{l}\text { With } 2 \text { other carbohydrates in } \\
\text { small tissue samples }\end{array}$ & [115] \\
\hline $\mathrm{GC}$ & FID & $\begin{array}{l}\text { DB- } 5 \text { capillary, } 15 \mathrm{~m} \times 0.53 \\
\text { mm (i.d.) }\end{array}$ & $\begin{array}{l}\text { Temp: Injection } 220^{\circ} \mathrm{C} \text {, detection } 300 \\
{ }^{\circ} \mathrm{C} \text {, flow } 9.7-9.9 \mathrm{ml} / \mathrm{min}\end{array}$ & $\begin{array}{l}\text { Ratios of lactulose/mannitol } \\
\text { excretion in urine }\end{array}$ & [116] \\
\hline HPLC & $\mathrm{RI}$ & $300 \times 6.5 \mathrm{~mm}$, Sugar Pak I & $\begin{array}{l}\text { Mobile phase: water, with calcium }(1 \\
\mathrm{ml} / \mathrm{l}, 50 \mathrm{~g} / \mathrm{l} \mathrm{Ca}-\mathrm{EDTA}) \text {, flow } 0.5 \\
\mathrm{ml} / \mathrm{min}, 85^{\circ} \mathrm{C}\end{array}$ & Lactulose/mannitol ratio & {$[117]$} \\
\hline GC & FID & $\begin{array}{l}\text { Capillary, } 17 \mathrm{~m} \times 0.25 \mathrm{~mm} \text { i.d., } \\
\text { coating, } 0.25 \mu \mathrm{m} \mathrm{OV}-17 \text {, fused } \\
\text { silica }\end{array}$ & $\begin{array}{l}\text { Detector and injector at } 300{ }^{\circ} \mathrm{C} \text {. The } \\
\text { oven temperature programmed } 120 \text { to } \\
140^{\circ} \mathrm{C} \text {. }\end{array}$ & Lactulose-mannitol pair in urine & [118] \\
\hline GC & MS & $\begin{array}{l}\text { Capillary, } 30 \mathrm{~m} \times 0.25 \mathrm{~mm} \\
\text { fused silica- } 50 \% \text { phenyl, } 50 \% \\
\text { methyl polysiloxane, } 0.25 \mu \mathrm{m} \\
\text { film thickness }\end{array}$ & Injector temp $250^{\circ} \mathrm{C}$, oven $150^{\circ} \mathrm{C}$ & $\begin{array}{l}\text { In CSF and plasma with } 7 \text { other } \\
\text { moieties; } 1,5 \text {-anhydrosorbitol, } \\
\text { ribitol, arabitol, myo-inositol, } \\
\text { xylitol, galactitol and. sorbitol }\end{array}$ & [119] \\
\hline GLC & FID & $\begin{array}{l}\text { Capillary, } 60 \mathrm{~m} \times 0.32 \mathrm{~mm} \text { ID. } \\
\text { fused silica }\end{array}$ & $\begin{array}{l}\text { Detector temperature, } 350{ }^{\circ} \mathrm{C} \text {; injector } \\
\text { temperature, } 250{ }^{\circ} \mathrm{C} \text {; } \\
\text { Min flow-rate was } 75 \mathrm{ml} / \mathrm{min}\end{array}$ & Urinary lactulose and mannitol & {$[120]$} \\
\hline HPLC & $\begin{array}{l}\text { Ampero } \\
\text { metry }\end{array}$ & $4 \mathrm{~mm}$ ID $\times 50 \mathrm{~mm}$, Dionex & Mobile phase: $500 \mathrm{mmol}, 0.4 \mathrm{ml} / \mathrm{min}$ & $\begin{array}{l}\text { Xylitol, sorbitol and D-Mannitol } \\
\text { in foods }\end{array}$ & {$[121]$} \\
\hline HPLC & $\begin{array}{l}\text { Ampero } \\
\text { metry }\end{array}$ & $\begin{array}{l}250 \times 4.0 \mathrm{~mm} \text { I.D., } 8.5 \mu \mathrm{m} \\
\text { particle size, anion exchange }\end{array}$ & $\begin{array}{l}480 \mathrm{mmol} \mathrm{NaOH} \text {, flow-rate: } 0.4 \\
\mathrm{ml} / \mathrm{min} .\end{array}$ & $\begin{array}{l}\text { Lactulose/mannitol ratio in } \\
\text { urine }\end{array}$ & {$[122]$} \\
\hline HPLC & $\begin{array}{l}\text { Ampero } \\
\text { metry }\end{array}$ & $\begin{array}{l}\text { Carbopac, } 4 \times 250 \mathrm{~mm} \text {, Anion- } \\
\text { exchange }\end{array}$ & $\begin{array}{l}\text { Mobile Phase: } \mathrm{NaOH} 160 \text { and } \\
\mathrm{Zn}\left(\mathrm{CH}_{3} \mathrm{CO}_{2}\right)_{2} 0.196 \mathrm{mmol} / 1 \text {, flow } 1.0 \\
\mathrm{ml} / \mathrm{min}\end{array}$ & $\begin{array}{l}\text { Mannitol and disaccharides in } \\
\text { serum }\end{array}$ & {$[123]$} \\
\hline HPLC & $\begin{array}{l}\text { Ampero } \\
\text { metry }\end{array}$ & $\begin{array}{l}\text { Anion exchange-Dionex PA-10 } \\
\text { and PA-100 }\end{array}$ & $\begin{array}{l}\text { Mobile phase: } \mathrm{NaOH}(0.1 \mathrm{M}) \text {; flow, } 1.0 \\
\mathrm{ml} \mathrm{min}^{-1}\end{array}$ & $\begin{array}{l}\text { Mannitol and lactulose-human } \\
\text { urine }\end{array}$ & {$[124]$} \\
\hline HPLC & $\begin{array}{l}\text { Photomet } \\
\text { ry }\end{array}$ & $\begin{array}{l}\text { Supelco TPR-100, } 15 \mathrm{~cm} \times 4.6 \\
\text { mm I. D }\end{array}$ & $\begin{array}{l}\text { Mobile phase: } 2 \mathrm{mmol} \mathrm{Na} \text { molybdate } \\
\text { and } 50 \mathrm{mmol} \mathrm{HNO}_{3}(\mathrm{pH} 1.4) \text { flow } 0.8 \\
\mathrm{ml} / \mathrm{min}, 600 \mathrm{~nm} .\end{array}$ & $\begin{array}{l}\text { D-mannitol and D-sorbitol in } \\
\text { foodstuffs }\end{array}$ & {$[125]$} \\
\hline GC & MS & $\begin{array}{l}30 \mathrm{~m} \times 30.25 \mathrm{~mm} \text { I. D, HP- } 5 \\
\text { fused-silica capillary, coating- } \\
0.25 \mu \mathrm{m} 5 \% \text { phenyl methyl } \\
\text { silicone }\end{array}$ & $\begin{array}{l}\text { Both injection port and transfer line } \\
280^{\circ} \mathrm{C} \text {, column head pressure } 50 \mathrm{kPa} \text {. } \\
\text { Ionization nrg } 70 \mathrm{eV} \text {. }\end{array}$ & In human brain tissues & [126] \\
\hline HPLC & UV & $\begin{array}{l}\mathrm{C}_{18}(250 \mathrm{~mm} \times 3: 0 \mathrm{~mm} \text { i.d., } 5 \\
\mu \mathrm{m}\end{array}$ & $\begin{array}{l}75 \% \text { ACN-water }(0.05 \% \text { TFA, } 0.05 \% \\
\text { heptafluoro-n-butyric acid and } 0.1 \% \\
\text { triethylamine), } 0.5 \mathrm{ml} / \mathrm{min} ; \lambda=260 \mathrm{~nm}\end{array}$ & With glycerol in human tissues & [127] \\
\hline HPLC & RI & $\begin{array}{l}\text { Amino column Nucleosil } 250 \times \\
4.6 \mathrm{~mm} \text { id }\end{array}$ & $\begin{array}{l}\text { Mobile Phase: ACN: } \mathrm{H}_{2} \mathrm{O}(7: 3, \mathrm{v} / \mathrm{v}), 1 \\
\mathrm{ml} / \mathrm{min}\end{array}$ & With lactulose in urine & [128] \\
\hline HILIC & ELSD & $\begin{array}{l}250 \mathrm{~mm} \times 4.6 \mathrm{~mm} \text { ID, } 5 \mu \mathrm{m}, \mathrm{Gel} \\
\text { Amide } 80\end{array}$ & $\begin{array}{l}\text { Mobile Phase: } 75: 25 \text { (ACN: } 0.1 \% \text { TFA } \\
\text { in } \mathrm{H}_{2} \mathrm{O} \text { ), flow } 1.0 \mathrm{ml} / \mathrm{min}\end{array}$ & In pharmaceutical formulations & [129] \\
\hline HPLC & MS/MS & $250 \mathrm{~mm} \times 2.1 \mathrm{~mm}, 5 \mu \mathrm{m}$ & $\begin{array}{l}\text { Mobile Phase: } 75 \% \text { ACN } / 25 \% 5 \mathrm{mmol} \\
\text { of ammonium acetate in } \mathrm{H}_{2} \mathrm{O} \text { to } 40 \% \\
\text { ACN } / 60 \% 5 \mathrm{mmol} \text { of ammonium } \\
\text { acetate in } \mathrm{H}_{2} \mathrm{O}(\mathrm{pH}=6.84) 10 \mathrm{~min}\end{array}$ & $\begin{array}{l}\text { With lactulose and sucrose in } \\
\text { human urine }\end{array}$ & {$[130]$} \\
\hline
\end{tabular}




\begin{tabular}{|c|c|c|c|c|c|}
\hline HPLC & RI & $\begin{array}{l}\text { Amino column } \\
(250 \times 4.6 \mathrm{~mm}, 5 \mu \mathrm{m})\end{array}$ & $\begin{array}{l}\text { Mobile Phase: ACN: } \mathrm{H}_{2} \mathrm{O}(86: 14) 1.0 \\
\mathrm{ml} / \mathrm{min}\end{array}$ & $\begin{array}{l}\text { Mannitol in Bailing } \\
\text { capsules }\end{array}$ & {$[131]$} \\
\hline HPLC & CAD & $\begin{array}{l}\text { Amino, packing, } 5 \mu \mathrm{m} \text { shell } \\
\text { particles }(4.6 \times 250 \mathrm{~mm})\end{array}$ & Mobile Phase: (Gradient) $\mathrm{ACN}$ and $\mathrm{H}_{2} \mathrm{O}$ & With 7 other carbohydrates & [132] \\
\hline HPLC & PAD & $\begin{array}{l}\text { Anion exchange column } \\
\text { (Pellicular resin), } 250 \times 4.0 \mathrm{~mm} \\
\text { i.d. Temperature } 30{ }^{\circ} \mathrm{C}\end{array}$ & $\begin{array}{l}\text { Mobile Phase: } 480 \mathrm{mmol} / \mathrm{l} \mathrm{NaOH} \text {, flow } \\
0.4 \mathrm{ml} / \mathrm{min}\end{array}$ & Lactulose: mannitol ratio & [133] \\
\hline LC & MS-MS & $\begin{array}{l}150 \times 2.1 \mathrm{~mm} 100 \mathrm{~A} \\
\text { aminopropylsilane Restek } \\
\text { column, } 3 \mu \mathrm{m} \text {, temperature } 40 \\
{ }^{\circ} \mathrm{C}\end{array}$ & $\begin{array}{l}\text { Mobile Phase: (Gradient) 0.1\% } \\
\text { HCOOH: ACN, flow } 0.30 \mathrm{ml} / \mathrm{min}\end{array}$ & & \\
\hline GC & FID & $\begin{array}{l}\text { Capillary, } 30 \mathrm{~m} \times 0.25 \mathrm{~mm} \\
\text { (HP-5MS) }\end{array}$ & $\begin{array}{l}\text { Mobile Phase: EtOH: } \mathrm{H}_{2} \mathrm{O}(80: 20) \text {, } \\
\text { Injector } 280^{\circ} \mathrm{C} \text {, detector } 300{ }^{\circ} \mathrm{C}\end{array}$ & $\begin{array}{l}\text { With myo-inositol and sorbitol } \\
\text { in roots of olive tree }\end{array}$ & [134] \\
\hline IC & PAD & Column: $4 \mathrm{~mm} \times 250 \mathrm{~mm}$ & Mobile Phase: $480 \mathrm{mmol} \mathrm{NaOH}$ eluent & $\begin{array}{l}\text { Mannitol, sorbitol and glucose- } \\
\text { containing powders }\end{array}$ & [135] \\
\hline LC & MS/MS & $\begin{array}{l}\mathrm{C}_{18} \text { column }(2.1 \mathrm{~mm} \times 100 \mathrm{~mm} \text {, } \\
3.5 \mu \mathrm{m})\end{array}$ & $\begin{array}{l}\text { Mobile Phase: (Gradient) } 10 \mathrm{mmol} \text { aq. } \\
\mathrm{NH}_{4} \mathrm{HCO}_{2} \text { buffer (pH 3.5): ACN. Flow: } \\
0.4 \mathrm{ml} / \mathrm{min}\end{array}$ & In human urine with glycerol & [136] \\
\hline LC & MS/MS & $\begin{array}{l}\text { Amino column, } 150 \times 2 \mathrm{~mm}, 5 \\
\mu \mathrm{m} 100 \mathrm{~A}\end{array}$ & $\begin{array}{l}\text { Mobile Phase: } \mathrm{ACN}: \mathrm{H}_{2} \mathrm{O} \text {, flow } 300 \\
\mu \mathrm{l} / \mathrm{min}\end{array}$ & Urinary lactulose and mannitol & [137] \\
\hline HPLC & RI & $\begin{array}{l}\text { Sugar SP0810 }(300 \mathrm{~mm} \times 8.0 \\
\text { mm i.d.) column }\end{array}$ & Mobile Phase: $\mathrm{H}_{2} \mathrm{O}$, flow $0.5 \mathrm{ml} / \mathrm{min}$ & $\begin{array}{l}\text { In dessert foods with erythritol, } \\
\text { sorbitol, isomalt and xylitol }\end{array}$ & [138] \\
\hline UPLC & MS/MS & Amide $1.7 \mu \mathrm{m}, 2.1 \times 50 \mathrm{~mm}$ & $\begin{array}{l}\text { Mobile Phase: (Gradient) ACN and } \\
\mathrm{H}_{2} \mathrm{O} \text {, flow } 200 \mu \mathrm{l} / \mathrm{min}\end{array}$ & $\begin{array}{l}\text { Urinary lactulose/mannitol } \\
\text { ratio }\end{array}$ & [139] \\
\hline HPAE & PAD & $\begin{array}{l}\text { Anion-exchange column } \\
(4 \times 250 \mathrm{~mm})\end{array}$ & $\begin{array}{l}\text { Mobile Phase: } 450 \mathrm{mmol} \mathrm{NaOH} \text {, flow } \\
0.4 \mathrm{ml} / \mathrm{min}\end{array}$ & $\begin{array}{l}\text { Urinary lactulose/mannitol } \\
\text { ratio }\end{array}$ & [140] \\
\hline LC & MS/MS & $\begin{array}{l}\text { Restek Ultramino } 150 \times 2.1 \\
\text { mm (100A } \\
\text { aminopropylsilane), } 3 \mu \mathrm{m}\end{array}$ & $\begin{array}{l}\text { Mobile Phase: (Gradient) } 0.1 \% \\
\mathrm{HCOOH} \text { and ACN }(+0.1 \% \mathrm{HCOOH}) \\
\text { flow } 0.30 \mathrm{ml} / \mathrm{min}\end{array}$ & & \\
\hline LC & MS/MS & $\begin{array}{l}\text { Amide }(2.1 \mathrm{~mm} \times 50 \mathrm{~mm} \text {, } \\
\text { particle size } 1.7 \mu \mathrm{m})\end{array}$ & $\begin{array}{l}\text { Mobile Phase: ACN: } \mathrm{H}_{2} \mathrm{O}: \mathrm{NH}_{4} \mathrm{OH} \\
(73: 27: 0.1, \mathrm{v} / \mathrm{v}), 0.2 \mathrm{ml} / \mathrm{min} .\end{array}$ & $\begin{array}{l}\text { Nonradiolabeled } \\
{\left[{ }^{13} \mathrm{C}_{6}\right] \text { mannitol }}\end{array}$ & [141] \\
\hline HPLC & MS/MS & Amino, $4.6 \times 250 \mathrm{~mm}, 5 \mu \mathrm{m}$ & $\begin{array}{l}\text { Mobile Phase: 70:30 ACN/10 mmol } \\
\mathrm{NH}_{4} \text {. Ac }\end{array}$ & $\begin{array}{l}\text { In Chinese medicine with } \\
\text { sorbitol and galactilol }\end{array}$ & [142] \\
\hline HPLC & RI & $\begin{array}{l}\text { Cation exchange resin }(\mathrm{Ca} \\
\text { form) }(0.3 \mathrm{~m} \text { long, } 7.8 \mathrm{~mm} \text { ID) } \\
{\left[85^{\circ} \mathrm{C} \pm 1^{\circ} \mathrm{C}\right]}\end{array}$ & Mobile Phase: $\mathrm{H}_{2} \mathrm{O}, 0.5 \mathrm{ml} / \mathrm{min}$ & Bulk & [143] \\
\hline HPLC & RI & $\begin{array}{l}(4 \times 25) \mathrm{mm}, \mathrm{L} 19 \text { packing, } 9 \\
\mu \mathrm{m} \text {, between } 30-85^{\circ} \mathrm{C} \pm 2{ }^{\circ} \mathrm{C}\end{array}$ & Mobile Phase: $\mathrm{H}_{2} \mathrm{O}, 0.5 \mathrm{ml} / \mathrm{min}$ & Bulk & [144] \\
\hline HPLC & RI & $\begin{array}{l}30 \mathrm{~cm} \times 7.8 \mathrm{~mm}, \mathrm{~mm} \text { packed } \\
\text { with strong cation exchange } \\
\text { resin (Ca form) } 9 \mu \mathrm{m}\end{array}$ & Mobile phase: $\mathrm{H}_{2} \mathrm{O}, 0.5 \mathrm{ml} / \mathrm{min}$ & Bulk & [145] \\
\hline GC & FID & $\begin{array}{l}6 \mathrm{ft} \times 32 \mathrm{~mm} \text { I.D. } 1 / 4 \mathrm{in} \text {, Glass } \\
\text { packed column }\end{array}$ & $\begin{array}{l}\text { Temperature: Detector, } 280^{\circ} \mathrm{C} \text {, } \\
\text { injector } 250^{\circ} \mathrm{C} \text {, initial column temp } \\
220^{\circ} \mathrm{C} . \mathrm{N}_{2} \text { (carrier gas) flow rate } 30 \\
\mathrm{ml} / \mathrm{min}\end{array}$ & Lactose Mannitol ratio in urine & [146] \\
\hline GC & FID & $\begin{array}{l}\text { Fused } 15 \mathrm{~m} \times 530 \mathrm{~mm} \text {, I.D. silica } \\
\text { capillary column }\end{array}$ & $\begin{array}{l}220^{\circ} \mathrm{C} \text { detector and injector } \\
\text { temperatures. He (carrier gas) } 10 \\
\mathrm{ml} / \mathrm{min}\end{array}$ & & \\
\hline HPLC & ELSD & $\begin{array}{l}\mathrm{C}_{18}, 300 \mathrm{~mm} \times 6.5 \mathrm{~mm} \text { i.d.) } 10 \\
\mu \mathrm{m}\end{array}$ & Mobile Phase: water, $0.5 \mathrm{ml} / \mathrm{min}$ & Lactulose and mannitol in urine & [147] \\
\hline HPLC & RI & $\begin{array}{l}\text { CHO- } 620 \text { cation-exchange } \\
\text { column, Temp } 85^{\circ} \mathrm{C}\end{array}$ & Mobile Phase: water, $0.4 \mathrm{ml} / \mathrm{min}$ & $\begin{array}{l}\text { With } 5 \text { other sugars in human } \\
\text { urine }\end{array}$ & [148] \\
\hline
\end{tabular}

\section{Other methods}

The alpha and delta polymorphs of mannitol are common contaminants and the beta form is usually used in pharmaceutical preparations. FTRaman spectroscopy was utilized for quantifying beta form in the presence of a mixture of beta and delta mannitol [149].

A method based on CE equipped with electrochemical detection without derivatization has been developed for sucrose, glucose, mannitol, and fructose estimation in Ligustrum lucidum [150].

Another CE method was developed to detect lactulose and mannitol in urine after oral administration to estimate the intestinal permeability in type I diabetes patients [151].

The CE method for the determination of xylitol, sorbitol, and mannitol as anionic borate-polyol complexes is also performed. The selected wavelength was $215 \mathrm{~nm}$ [152].

Quantitative estimation of polymorphs of mannitol by FT-Raman spectroscopic data using both partial least-squares (PLS) regression as well as artificial neural networks (ANNs) performed by Braun et al. [153]

Since mannitol is excreted unchanged through urine in normal pathological conditions, thus for sugar intake can be used as a reference compound. One of the recent research is the determination of sucrose, lactulose, and mannitol in urine by using NMR spectroscopy [154].

\section{DISCUSSION}

The history of analysis of Mannitol from 1970 onwards started with Gas-liquid chromatography methods. Gas Chromatography (GC) has incontestable to be a flexible and sensitive analytical technique for comprehensive chemical characterization of food samples [155]. The analytical methods discussed here are also presented in fig. 10.

Various available methods are based on this technique based on either MS or FID detector systems. The FID detectors are commonly used for the analysis with the GC system. The utilization of these two detectors may be due to their proven application in food authenticity. Mannitol is a widely distributed sugar alcohol in nature, and FID detectors are sensitive and have a wide linear range making it suitable for determination [156]. Whereas MS detectors overcome the problem related to detection limits because of their high resolution and specificity [157].

The same pattern was observed in the case of mannitol determination based on GC. Methods based on FID were mostly 
applied for the determination of other sugars or lactose-mannitol ratio, whereas complex samples, e. g., plasma or serum, GC system based on MS detectors.

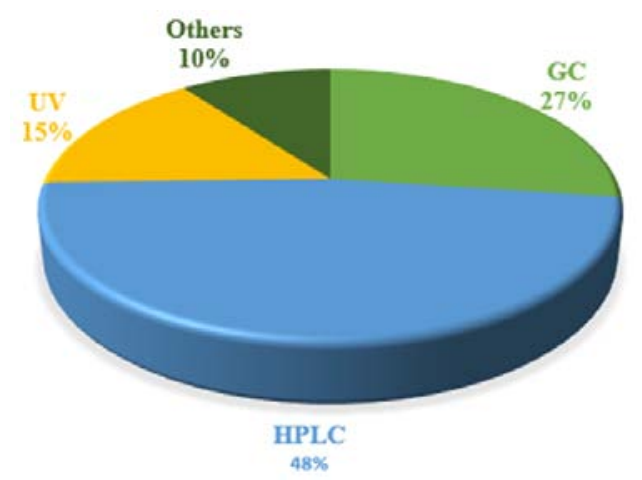

Fig. 10: Available analytical methods for mannitol (1970-till date)

Spectrophotometry is a fundamental technique in many areas of science, with many applications and uses [158]. Various spectrophotometry methods are available for analysis, particularly the lactose-mannitol ratio in urine. However, chromatography methods are also available for the same applications but spectrophotometry is an easy and economical technique. The only factors that limit the usage of the latter are sensitivity and the capability to analyze complex biological samples.

The requirement for better strategies for analysis is normal because of new requests like information and the prerequisites to break down a variety of new types of samples. Many older HPLC techniques can be altered and improved to incorporate new prerequisites and additionally new capacities [159]. Basic measurements for analytical scale columns are in the range of 10-30 cm long and 4-10 $\mathrm{mm}$ i. d and smaller dimensions are most efficient and have the advantage of the speed and minimal solvent consumption [160].

The method developed by Yada et al. [121] published in the year 1996 also utilizes a short column based on amperometry detection, as claimed by the research group, is about 1000 times more sensitive than all previously HPLC equipped with RI detectors and GC-FID methods. The UPLC method developed by Gervasoni and coresearchers [139] used a column (50 mm length) that separated mannitol and lactulose in just $5 \mathrm{~min}$. Another recent UPLC method for labeled isotopes of mannitol and sucrose simultaneously in brain and plasma with a total run time of $6 \mathrm{~min}$ [141].

The intestinal permeability (IP) test is a reasonable, precise strategy for assessing the integrity of the gastrointestinal mucosa without utilizing invasive strategies such as radiology or endoscopy [161]. The utilization of 51Cr EDTA does not generally ensure dependable outcomes since the utilization of a solitary molecule can recognize changes not identified with the penetrability alterations [162].

The assessment of intestinal permeability dependent on the evaluated assimilation of two sugars of various sizes gives more data and higher sensitivity than utilizing solitary sugar. In a physiological condition, the rate of absorption is about $10 \%$ for mannitol and under $1 \%$ for lactulose. The deficiency of mucosal integrity ought to cause an increment of lactulose retention with a subsequent increment of lactulose-mannitol proportion in the sample of urine [163].

Diverse laboratory methods have been proposed for the measurement of mannitol and lactulose in urine, such as spectrophotometric [102] and enzymatic [69, 97-9, 101-3] methods, gas chromatography $[116,118,120,146]$, and high-performance liquid chromatography [117, 122-23, 128, 133, 137, 139-40, 147].

One of the recent trends in Green Analytical Chemistry is developing straightforward and low-cost ways for the qualitative and/or quantitative determination of various analytes [164]. The aim is to minimize the usage of chemicals and overall energy consumption, analytical waste management, and safety of analysts [165].

In the case of mannitol, various analytical methods reported are presented in the section above. Various spectrophotometry, chromatography, electroanalytical and other methods are available for determination. In most of the analytical methods, water plays an important role as a diluent or mobile phase because of the solubility of mannitol in it. A few chromatography methods are based on nonaqueous phases in some proportion. Proper automation, high pressure, and reducing column sizes and speed maybe some of the approaches for the development of better eco-friendly analytical methods.

\section{CONCLUSION}

Mannitol is a drug as well as one of the popular excipients for many formulations. This is poorly absorbed from the intestine and can be used as a sugar substitute for diabetic patients because of its sweet taste. There are many spectrophotometry and chromatographic methods developed and validated for the determination of mannitol in different matrices. The spectrophotometry and chromatography methods are given in Tables 4 and 5 respectively. Mannitol is an aliphatic compound without any aromatic rings which cannot be easily measured under the UV spectrophotometry method. Most of the spectrophotometry methods developed are based on the enzymatic conversion of mannitol into a suitable form for successful determinations. These methods are tedious, requiring additional culture of enzyme, and are time-consuming. Mannitol can be determined by using any other chemical reactions, e. g., coupling with any dye with simple ionic interactions like in acid dye methods or addition of aromatic moiety like benzene in its structure, making it feasible to be identified under UV spectrophotometers. Although some of the chromatographic methods, e. g., HPLC methods, are based on the aqueous mobile phase, these methods are expensive, time-consuming, and require a specific type of columns. In addition, a few reported methods use non-aqueous phases also in mobile phases in some proportion. Researchers should also think about the utilization of green analytical chemistry for mannitol for faster, easy, economical analytical methods safer to analysts as well as to the environment.

\section{ACKNOWLEDGEMENT}

The authors acknowledge the support given by KIET Group of Institutions for giving support in providing facilities for the study. Also, we are thankful to Shiv Nadar University, Greater Noida for providing NMR spectra of Mannitol included in the manuscript.

\section{ABBREVIATIONS}

United States Pharmacopoeia (USP), Indian Pharmacopoeia (IP) and British Pharmacopoeia (BP), Inhaled dry powder mannitol (IDPM), Intradialytic hypotension (IDH), Mannitol Dehydrogenase (MDH), Formate Dehydrogenase (FDH), Nicotinamide adenine dinucleotide (NAD), Nicotinamide adenine dinucleotide phosphate (NADP), Generally Recognized as Safe (GRAS), Central Nervous System (CNS), Dry powder inhaler (DPI), octadecyl (OD)

\section{FUNDING}

Nil

\section{AUTHORS CONTRIBUTIONS}

All authors have contributed equally.

\section{CONFLICT OF INTERESTS}

No potential conflict of interest was reported by the authors.

\section{REFERENCES}

1. Mannitol. Available from: https://pubchem.ncbi.nlm.nih.gov/ compound/Mannitol. [Last accessed on 10 Apr 2021]

2. Martau GA, Coman V, Vodnar DC. Recent advances in the biotechnological production of erythritol and mannitol. Crit Rev Biotechnol 2020;40:608-22. 
3. Jivraj II, Martini LG, Thomson CM. An overview of the different excipients useful for the direct compression of tablets. Pharm Sci Technol Today 2000;3:58-63.

4. The Merck Index: an encyclopedia of chemicals, drugs, and biologicals. 14th Ed. Edited by Maryadele J. O'Neil, Patricia E Heckelman, Cherie B Koch, Kristin J Roman. Merck J Am Chem Soc 2007;129:2197.

5. Rajagopalan V, Gupta N. Osmotherapy. In pharmacology in clinical neurosciences; Springer Singapore: Singapore; 2020. p. 257-64.

6. Peng $\mathrm{Y}$, Liu X, Wang A, Han R. The effect of mannitol on intraoperative brain relaxation in patients undergoing supratentorial tumor surgery: study protocol for a randomized controlled trial. Trials 2014;15:165.

7. Montazeri K, Soltani H, Varposhti MR, Mohajeri L. Comparison of preoperative infusion of magnesium sulfate and mannitol on intraocular pressure changes in patients undergoing phacoemulsification surgery. Adv Biomed Res 2018;7:39.

8. Nissenson AR, Weston RE, Kleeman CR. Mannitol. West J Med 1979;131:277-84.

9. He Q Liu J, Liang J, Liu X, Li W, Liu Z, et al. Towards improvements for penetrating the blood-brain barrier-recent progress from a material and pharmaceutical perspective. Cells 2018;7:24.

10. Bellavance MA, Blanchette M, Fortin D. Recent advances in blood-brain barrier disruption as a CNS delivery strategy. AAPS J 2008;10:166-77.

11. Herraiz Hidalgo L, Alvarez Moreno E, Carrascoso Arranz J, Cano Alonso R, Martinez de Vega Fernandez V. Magnetic resonance enterography: review of the technique for the study of crohn's disease. Radiologia 2011;53:421-33.

12. Bilton D, Robinson P, Cooper P, Gallagher CG, Kolbe J, Fox H, et al. CF301 study investigators. Inhaled dry powder mannitol in cystic fibrosis: an efficacy and safety study. Eur Respir J 2011;38:1071-80.

13. Hurt K, Bilton D. Inhaled mannitol for the treatment of cystic fibrosis. Expert Rev Respir Med 2012;6:19-26.

14. Ruiz Rodriguez LG, Aller K, Bru E, De Vuyst L, Hebert EM, Mozzi F. Enhanced mannitol biosynthesis by the fruit origin strain Fructobacillus tropaeoli CRL 2034. Appl Microbiol Biotechnol 2017;101:6165-77.

15. Mannitol Market Projected To Be Worth \$418.3 Million By 2024 . Available from: https://www.grandviewresearch.com/pressrelease/global-mannitol-market. [Last accessed on $10 \mathrm{Apr} 2021]$

16. Persaud N, Jiang M, Shaikh R, Bali A, Oronsaye E, Woods H, et al. Comparison of essential medicines lists in 137 countries. Bull World Health Organ 2019;97:394-404C.

17. Walsh J, Cram A, Woertz K, Breitkreutz J, Winzenburg G, Turner $\mathrm{R}$, et al. European formulation initiative. Playing hide and seek with poorly tasting paediatric medicines: do not forget the excipients. Adv Drug Delivery Rev 2014;73:14-33.

18. Grembecka M. Sugar alcohols-their role in the modern world of sweeteners: a review. Eur Food Res Technol 2015;241:1-14.

19. Daraghmeh N, Rashid I, Al Omari MM, Leharne SA, Chowdhry BZ, Badwan A. Preparation and characterization of a novel coprocessed excipient of chitin and crystalline mannitol. AAPS PharmSciTech 2010;11:1558-71.

20. Al-Khattawi A, Koner J, Rue P, Kirby D, Perrie Y, Rajabi Siahboomi A, et al. A pragmatic approach for engineering porous mannitol and mechanistic evaluation of particle performance. Eur J Pharm Biopharm 2015;94:1-10.

21. Crain SM, Peterson ER. Selective innervation of target regions within fetal mouse spinal cord and medulla explants by isolated dorsal root ganglia in organotypic co-cultures. Brain Res 1981;254:341-62.

22. Ohrem HL, Schornick E, Kalivoda A, Ognibene R. Why is mannitol becoming more and more popular as a pharmaceutical excipient in solid dosage forms? Pharm Dev Technol 2014;19:257-62.

23. Handbook of Pharmaceutical Excipients. $7^{\text {th }}$ Edition. Pharm Dev Technol; 2013. p. 544.

24. Birinyi Strachan LC, Davies MJ, Lewis RJ, Nicholson GM. Neuroprotectant effects of iso-osmolar $D$-mannitol to prevent pacific ciguatoxin-1 induced alterations in neuronal excitability: a comparison with other osmotic agents and free radical scavengers. Neuropharmacology 2005;49:669-86.
25. Bernstein LM, Ivy AC. Inositol and mannitol hexanitrates in hypertension management. Circulation 1955;12:353-60.

26. Shawkat H, Westwood MM, Mortimer A. Mannitol: a review of its clinical uses. Contin Educ Anaesth Crit Care Pain 2012;12:82-5.

27. Kavanagh O, Hogan F, Murphy C, Croker D, Walker G. Formulating a stable mannitol infusion while maintaining hyperosmolarity. Pharmaceutics 2020;12:187.

28. Perry RH, Green DW, Maloney JO. Perry's chemical engineers' handbook. 7th ed. McGraw-Hill, New York; 1997.

29. Godswill AC. Sugar alcohols: chemistry, production, health concerns and nutritional importance of mannitol, sorbitol, xylitol, and erythritol. Int J Adv Acad Res Sci Technol Eng 2017;3:31-66.

30. Deis RC, Kearsley MW. Sorbitol and mannitol. In: Sweeteners and sugar alternatives in food technology, Wiley-Blackwell: Oxford UK; 2012. p. 331-46.

31. Kumar MV. Formulation and evaluation of meclizine $\mathrm{HCl}$ orally dispersible tablets by using natural super disintegrants. Int J Pharma Sci Res 2016;2:53-80.

32. BeMiller JN. Carbohydrates. In: Kirk-othmer encyclopedia of chemical technology; John Wiley and Sons, Inc: Hoboken NJ, USA; 2004.

33. Bolhuis GK, Rexwinkel EG, Zuurman K. Polyols as filler-binders for disintegrating tablets prepared by direct compaction. Drug Dev Ind Pharm 2009;35:671-7.

34. Tavakkoli F. Review of the role of mannitol in the therapy of children. 18 ${ }^{\text {th }}$ Expert Comm Sel Use Essent Med; 2011. p. 1-43.

35. Winkler SR, Munoz Ruiz L. Mechanism of action of mannitol. Surg Neurol 1995;43:59.

36. Oreskovic D, Marakovic J, Varda R, Rados M, Jurjevic I, Klarica M. New insight into the mechanism of mannitol effects on cerebrospinal fluid pressure decrease and craniospinal fluid redistribution. Neuroscience 2018;392:164-71.

37. Tenny S, Patel R, Thorell W. Mannitol. In: StatPearls. Treasure Island (FL): StatPearls Publishing; 2021. Available from: https://www.ncbi.nlm.nih.gov/books/NBK470392/. [Last accessed on 24 Nov 2020]

38. Mannitol. In Meyler's Side Effects of Drugs; Elsevier; 2016. p. 744-51.

39. Nguyen T, Veltchev K, Nguyen TV. Mannitol role in the management of intradialytic hypotension. Am J Ther 2020;27:e450-54.

40. Mc Causland FR, Claggett B, Sabbisetti VS, Jarolim P, Waikar SS Hypertonic mannitol for the prevention of intradialytic hypotension: a randomized controlled trial. Am J Kidney Dis 2019;74:483-90.

41. Ghoreishi SM, Sharifi S. Modeling of supercritical extraction of mannitol from plane tree leaf. J Pharm Biomed Anal 2001;24:1037-48.

42. Ghoreishi SM, Shahrestani RG. Subcritical water extraction of mannitol from olive leaves. J Food Eng 2009;93:474-81.

43. Soetaert $\mathrm{W}$, Vanhooren PT, VEJ. The production of mannitol by fermentation. In: Bucke C. (Eds) Carbohydrate Biotechnology Protocols. Methods in Biotechnology ${ }^{\mathrm{TM}}$ : Humana Press; 1999.

44. Von Weymarn N, Hujanen M, Leisola M. Production of $D$ mannitol by heterofermentative lactic acid bacteria. Process Biochem 2002;37:1207-13.

45. Zhang M, Gu L, Cheng C, Ma J, Xin F, Liu J, et al. Recent advances in microbial production of mannitol: utilization of low-cost substrates, strain development, and regulation strategies. World J Microbiol Biotechnol 2018;34:41.

46. Kulbe KD, Schwab U, Gudernatsch W. Enzyme-catalyzed production of mannitol and gluconic acid. Product recovery by various procedures. Ann N Y Acad Sci 1987;506:552-68.

47. Wichmann R, Wandrey C, Buckmann AF, Kula MR. Continuous enzymatic transformation in an enzyme membrane reactor with simultaneous $\mathrm{NAD}(\mathrm{H})$ regeneration. Biotechnol Bioeng 2000;67:791-804.

48. Park YC, Oh EJ, Jo JH, Jin YS, Seo JH. Recent advances in biological production of sugar alcohols. Curr Opin Biotechnol 2016;37:105-13.

49. Saha BC, Nakamura LK. Production of mannitol and lactic acid by fermentation with Lactobacillus intermedius NRRL B-3693. Biotechnol Bioeng 2003;82:864-71. 
50. Smiley KL, Cadmus MC, Liepins P. Biosynthesis of $D$-mannitol from D-glucose by Aspergillus Candidus. Biotechnol Bioeng 1967;9:365-74.

51. Saha BC, Nakamura LK. Production of mannitol and lactic acid by fermentation with Lactobacillus intermedius NRRL B-3693. Biotechnol Bioeng 2003;82:864-71.

52. Hendriksen HV, Mathiasen TE, Adler Nissen J, Frisvad JC, Emborg C. Production of mannitol by penicillium strains. J Chem Technol Biotechnol 2007;43:223-8.

53. Meng Q, Zhang T, Wei W, Mu W, Miao M. Production of mannitol from a high concentration of glucose by Candida parapsilosis SK26.001. Appl Biochem Biotechnol 2017;181:391-406.

54. Qin J, Zhou YJ, Krivoruchko A, Huang M, Liu L, Khoomrung S, et al. Modular pathway rewiring of Saccharomyces Cerevisiae enables high-level production of l-ornithine. Nat Commun 2015;6:8224.

55. Wisselink H, Weusthuis R, Eggink G, Hugenholtz J, Grobben G. Mannitol production by lactic acid bacteria: a review. Int Dairy J 2002;12:151-61.

56. Gaspar P, Neves AR, Ramos A, Gasson MJ, Shearman CA, Santos $\mathrm{H}$. Engineering lactococcus lactis for production of mannitol: high yields from food-grade strains deficient in lactate dehydrogenase and the mannitol transport system. Appl Environ Microbiol 2004;70:1466-74.

57. Helanto M, Aarnikunnas J, von Weymarn N, Airaksinen U, Palva A, Leisola M. Improved mannitol production by a random mutant of Leuconostoc pseudomesenteroides. J Biotechnol 2005;116:283-94.

58. Costenoble R, Adler L, Niklasson C, Liden G. Engineering of the metabolism of Saccharomyces cerevisiae for anaerobic production of mannitol. FEMS Yeast Res 2003;3:17-25.

59. Kaup B, Bringer Meyer S, Sahm H. Metabolic engineering of Escherichia coli: construction of an efficient biocatalyst for Dmannitol formation in a whole-cell biotransformation. Appl Microbiol Biotechnol 2004;64:333-9.

60. Kaup B, Bringer Meyer S, Sahm HD. Mannitol formation from D:-glucose in a whole-cell biotransformation with recombinant Escherichia coli. Appl Microbiol Biotechnol 2005;69:397-403.

61. Schafer A, Stein MA, Schneider KH, Giffhorn F. Mannitol dehydrogenase from Rhodobacter sphaeroides Si4:subcloning, overexpression in Escherichia coli and characterization of the recombinant enzyme. Appl Microbiol Biotechnol 1997;48:47-52.

62. Cassanelli M, Norton I, Mills T. Interaction of mannitol and sucrose with gellan gum in freeze-dried gel systems. Food Biophys 2018;13:304-15.

63. Saha BC, Racine FM. Biotechnological production of mannitol and its applications. Appl Microbiol Biotechnol 2011;89:879-91.

64. Patra F, Tomar SK, Arora S. Technological and functional applications of low-calorie sweeteners from lactic acid bacteria. J Food Sci 2009; 74:R16-23.

65. Andre P, Villain F. Free radical scavenging properties of mannitol and its role as a constituent of hyaluronic acid fillers: a literature review. Int J Cosmet Sci 2017;39:355-60.

66. Rapoport SI. Advances in osmotic opening of the blood-brain barrier to enhance CNS chemotherapy. Expert Opin Investig Drugs 2001;10:1809-18.

67. Li XY, Gilmour PS, Donaldson K, MacNee W. Free radical activity and pro-inflammatory effects of particulate air pollution (PM10) in vivo and in vitro. Thorax 1996;51:1216-22.

68. Eggleston $\mathrm{G}$, Legendre $\mathrm{B}$, Tew $\mathrm{T}$. Indicators of freeze-damaged sugarcane varieties which can predict processing problems. Food Chem 2004;87:119-33.

69. Eggleston G, Harper W. Determination of sugarcane deterioration at the factory: development of a rapid, easy and inexpensive enzymatic method to measure mannitol. Food Chem 2006;98:366-72.

70. Su W, Jia N, Li H, Hao H, Li C. Polymorphism of D-mannitol: crystal structure and the crystal growth mechanism. Chinese J Chem Eng 2017;25:358-62.

71. Yoshinari T, Forbes RT, York P, Kawashima Y. Moisture induced polymorphic transition of mannitol and its morphological transformation. Int J Pharm 2002;247:69-77.

72. Lyu F, Liu JJ, Zhang Y, Wang XZ. Combined control of morphology and polymorph in spray drying of mannitol for dry powder inhalation. J Cryst Growth 2017:467:155-61.
73. Lee EH. A practical guide to pharmaceutical polymorph screening and selection. Asian J Pharm Sci 2014;9:163-75.

74. Tao J, Jones KJ, Yu L. Cross-nucleation between d-mannitol polymorphs in seeded crystallization. Cryst Growth Des 2007; 7:2410-14.

75. Besenhard MO, Neugebauer P, Scheibelhofer O, Khinast JG. Crystal engineering in continuous plug-flow crystallizers. Cryst Growth Des 2017;17:6432-44.

76. Kaminsky W, Glazer AM. Crystal optics of d-mannitol, $\mathrm{C}_{6} \mathrm{H}_{14} \mathrm{O}_{6}$ : Crystal growth, structure, basic physical properties, birefringence, optical activity, faraday effect, electro-optic effects and model calculations. Zeitschrift Fur Krist Cryst Mater 1997;212. DOI:10.1524/zkri.1997.212.4.283.

77. Cares Pacheco MG, Vaca Medina G, Calvet R, Espitalier F, Letourneau JJ, Rouilly A, et al. Physicochemical characterization of D-mannitol polymorphs: the challenging surface energy determination by inverse gas chromatography in the infinite dilution region. Int J Pharm 2014;475:69-81.

78. Hao H, Su W, Barrett M, Caron V, Healy AM, Glennon B. A calibration-free application of raman spectroscopy to the monitoring of mannitol crystallization and its polymorphic transformation. Org Process Res Dev 2010;14:1209-14.

79. Su W, Hao H, Glennon B, Barrett M. Polymorphic nucleation of d-mannitol in aqueous solution monitored with raman spectroscopy and FBRM. Cryst Growth Des 2013;13:5179-87.

80. Karthikeyan S, Nagasudhan N, Rogan AN, Senthilkumar A, Leelaprasad G. Transient numerical analysis on the melting characteristics of paraffin and d-mannitol phase change materials for latent thermal energy storage. Mater Today Proc 2021;45:6306-13.

81. Ward DR, Lathrop LB, Lynch MJ. Dissolution and compatibility considerations for the use of mannitol in solid dosage forms. J Pharm Sci 1969;58:1464-67.

82. Wang Z. Maillard reaction. In: Comprehensive organic name reactions and reagents; John Wiley and Sons, Inc.: Hoboken NJ, USA; 2010.

83. Chooluck K, Rojsanga $\mathrm{P}$, Phechkrajang C, Jaturanpinyo M. Bioanalytical method validation for determination of rosmarinic acid in simulated biological media using HPLC. Int J Appl Pharm 2021;13:110-3.

84. Pereira RN, Valente BR, Cruz AP, Foppa T, Murakami FS, Silva MAS. Thermoanalytical study of atenolol and commercial tablets. Latt Am J Pharm 2007;26:382-6.

85. Wesolowski M, Rojek B. Thermogravimetric detection of incompatibilities between atenolol and excipients using multivariate techniques. J Therm Anal Calorim 2013;113:169-77.

86. Tomassetti M, Catalani A, Rossi V, Vecchio S. Thermal analysis study of the interactions between acetaminophen and excipients in solid dosage forms and some binary mixtures. J Pharm Biomed Anal 2005;37:949-55.

87. Adkin DA, Davis SS, Sparrow RA, Huckle PD, Wilding IR. The effect of mannitol on the oral bioavailability of cimetidine. J Pharm Sci 1995;84:1405-9.

88. Late SG, Banga AK. Thermal and non-thermal methods to evaluate compatibility of granisetron hydrochloride with tablet excipients. Pharmazie 2008;63:453-8.

89. Joshi BV, Patil VB, Pokharkar VB. Compatibility studies between carbamazepine and tablet excipients using thermal and nonthermal methods. Drug Dev Ind Pharm 2002;28:687-94.

90. Agatonovic Kustrin S, Markovic N, Ginic Markovic M, Mangan M, Glass BD. Compatibility studies between mannitol and omeprazole sodium isomers. J Pharm Biomed Anal 2008;48:356-60.

91. Julio TA, Zamara IF, Garcia JS, Trevisan MG. Compatibility of sildenafil citrate and pharmaceutical excipients by thermal analysis and LC-UV. J Therm Anal Calorim 2013;111:2037-44.

92. Bertol CD, Cruz AP, Stulzer HK, Murakami FS, Silva MAS Thermal decomposition kinetics and compatibility studies of primaquine under isothermal and non-isothermal conditions. J Therm Anal Calorim 2010;102:187-92.

93. Raposo F, Ibelli Bianco C. Performance parameters for analytical method validation: controversies and discrepancies among numerous guidelines. TrAC Trends Anal Chem 2020;129:115913. 
94. Siddiqui MR, AlOthman ZA, Rahman N. Analytical techniques in pharmaceutical analysis: a review. Arab J Chem 2017;10:S1409-S1421.

95. Hammond JP. The use of spectrophotometry in the pharmaceutical industry. Exp Methods Phys Sci 2014;46:409-56.

96. Koko MYF, Mu W, Hassanin HAM, Zhang S, Lu H, Mohammed JK, et al. Archaeal hyperthermostable mannitol dehydrogenases: a promising industrial enzymes for d-mannitol synthesis. Food Res Int 2020;137:109638.

97. Blomquist $\mathrm{CH}$, Snyder BD, Niehaus WG. Improved enzymatic method for determining mannitol and its application to dog serum after mannitol infusion. J Clin Chem Clin Biochem 1981;19:139-43.

98. Blood J, Ingle AR, Allison N, Davies GR, Hill PG. Rapid enzymatic method for the measurement of mannitol in urine. Ann Clin Biochem 1991;28:401-6.

99. Berezenko S, Sturgeon RJ. The enzymic determination of dmannitol with mannitol dehydrogenase from agaricus bisporus. Carbohydr Res 1992;216:505-9.

100. Wingler A, Guttenberger M, Hampp R. Determination of mannitol in ectomycorrhizal fungi and ectomycorrhizas by enzymatic micro-assays. Mycorrhiza 1993;3:69-73.

101. Graefe H, Gutschow B, Gehring H, Dibbelt L. Sensitive and specific photometric determination of mannitol in human serum. Clin Chem Lab Med 2003;41:1049-55.

102. Zhang Y, Lee B, Thompson M, Glass R, Cama RI, Figueroa D, et al. Lactulose-mannitol intestinal permeability test in children with diarrhea caused by rotavirus and cryptosporidium. Diarrhea working group peru. J Pediatr Gastroenterol Nutr 2000;31:16-21.

103. Lunn PG, Northrop CA, Northrop AJ. Automated enzymatic assays for the determination of intestinal permeability probes in urine. 2. Mannitol. Clin Chim Acta 1989;183:163-70.

104. Hessels J, Snoeyink EJ, Platenkamp AJ, Voortman G, Steggink J, Eidhof HH. Assessment of intestinal permeability: enzymatic determination of urinary mannitol, raffinose, sucrose and lactose on hitachi analyzer. Clin Chem Lab Med 2003;41:33-8.

105. Hage DS. Chromatography and electrophoresis. In: Contemporary practice in clinical chemistry, Elsevier; 2019. p. 135-57.

106. Manius G, Mahn FP, Venturella VS, Senkowski BZ. GLC determination of sorbitol and mannitol in aqueous solutions. J Pharm Sci 1972;61:1831-5.

107. Sondack DL, Sondack DL. Determination of sorbitol and mannitol in pharmaceuticals by GLC of tris-n-butyldiboronate esters. J Pharm Sci 1975;64:128-9.

108. Servo C, Palo J, Pitkanen E. Gas chromatographic separation and mass spectrometric identification of polyols in human cerebrospinal fluid and plasma. Acta Neurol Scand 1977;56:104-10.

109. Daniels DH, Warner CR, Fazio T. Gas chromatographic determination of sorbitol, mannitol, and xylitol in chewing gum and sorbitol in mints. J Assoc Off Anal Chem 1982;65:588-91.

110. Vidal Valverde C, Martin Villa C, Olmedilla B. Improved separation of polyols and carbohydrates by high performance liquid chromatography. J Liq Chromatogr 1982;5:1941-6.

111. Samarco EC, Parente ES. Automated high pressure liquid chromatographic system for determination of mannitol, sorbitol, and xylitol in chewing gums and confections. J Assoc Off Anal Chem 1982;65:76-8.

112. Marunaka T, Matsushima E, Umeno Y, Minami Y. GLC-mass fragmentographic determination of mannitol and sorbitol in plasma. J Pharm Sci 1983;72:87-9.

113. Roboz J, Kappatos DC, Greaves J, Holland JF. Determination of polyols in serum by selected ion monitoring. Clin Chem 1984;30:1611-5.

114. Ali MS. Rapid quantitative method for the determination of dextrose, mannitol, and sorbitol in meat products by liquid chromatography. J Liq Chromatogr 1988;11:3271-81.

115. Eades DM, Williamson JR, Sherman WR. Rapid analysis of sorbitol, galactitol, mannitol and myoinositol mixtures from biological sources. J Chromatogr 1989;490:1-8.

116. Shippee RL, Johnson AA, Cioffi WG, Lasko J, LeVoyer TE, Jordan BS. Simultaneous determination of lactulose and mannitol in urine of burn patients by gas-liquid chromatography. Clin Chem 1992;38:343-5.

117. Willems D, Cadranel S, Jacobs W. Measurement of urinary sugars by HPLC in the estimation of intestinal permeability: evaluation in pediatric clinical practice. Clin Chem 1993;39:888-90.

118. Relva AMS, das Neves HJC, Ferreira MA. Determination of lactulose and mannitol in urine by HRGC. J High Resolut Chromatogr 1995;18:692-4.

119. Shetty HU, Holloway HW, Rapoport SI. Capillary gas chromatography combined with ion trap detection for quantitative profiling of polyols in cerebrospinal fluid and plasma. Anal Biochem 1995;224:279-85.

120. Martínez Augustin 0, Boza JJ, Romera JM, Gil A. A rapid gasliquid chromatography method for the determination of lactulose and mannitol in urine: clinical application in studies of intestinal permeability. Clin Biochem 1995;28:401-5.

121. Yada T, Tabuchi Y, Fujii M, Koh T, Tobimatsu Y, Hamasaki N, et al. Determination of xylitol, d-sorbitol and d-mannitol in food by anion-exchange chromatography with pulsed amperometric detection. Eisei Kagaku 1996;42:417-21.

122. Bao Y, Silva TM, Guerrant RL, Lima AM, Fox JW. Direct analysis of mannitol, lactulose and glucose in urine samples by highperformance anion-exchange chromatography with pulse amperometric detection. Clinical evaluation of intestinal permeability in human immunodeficiency virus infection. J Chromatogr B Biomed Appl 1996;685:105-12.

123. Cox MA, Iqbal TH, Cooper BT, Lewis KO. An analytical method for the quantitation of mannitol and disaccharides in serum: a potentially useful technique in measuring small intestinal permeability in vivo. Clin Chim Acta 1997;263:197-205.

124. Wring SA, Terry A, Causon R, Jenner WN. The electroanalysis of mannitol, xylose and lactulose at copper electrodes: voltammetric studies and bioanalysis in human urine by means of HPLC with electrochemical detection. J Pharm Biomed Anal 1998;16:1213-24.

125. Yamamoto A, Ohmi H, Matsunaga A, Ando K, Hayakawa K, Nishimura M. Selective determination of $d$-sorbitol and $d$ mannitol in foodstuffs by ion chromatography with polarized photometric detection. J Chromatogr A 1998;804:305-9.

126. Kiyoshima A, Kudo K, Hino Y, Ikeda N. Sensitive and simple determination of mannitol in human brain tissues by gas chromatography-mass spectrometry. J Chromatogr B Biomed Sci Appl 2001;758:103-8.

127. Kiyoshima A, Kudo K, Nishida N, Ikeda N. HPLC simultaneous determination of glycerol and mannitol in human tissues for forensic analysis. Forensic Sci Int 2002;125:127-33.

128. Liu H, Zhang S, Yu A, Qu L, Zhao Y, Huang H, et al. Studies on intestinal permeability of cirrhotic patients by analysis lactulose and mannitol in urine with HPLC/RID/MS. Bioorg Med Chem Lett 2004;14:2339-44.

129. Risley DS, Yang WQ, Peterson JA. Analysis of mannitol in pharmaceutical formulations using hydrophilic interaction liquid chromatography with evaporative light-scattering detection. J Sep Sci 2006;29:256-64.

130. Kubica P, Kot Wasik A, Wasik A, Namiesnik J, Landowski P. Modern approach for determination of lactulose, mannitol and sucrose in human urine using HPLC-MS/MS for the studies of intestinal and upper digestive tract permeability. J Chromatogr B: Anal Technol Biomed Life Sci 2012;907:34-40.

131. Liu W, Xiao X, Song Z, Cheng X, Wei F, Liu Y, et al. Research on determination of mannitol in bailing capsules by HPLC-RID instead of titration. Chinese J Pharm Anal 2014;34:159-62.

132. Grembecka M, Lebiedzinska A, Szefer P. Simultaneous separation and determination of erythritol, xylitol, sorbitol, mannitol, maltitol, fructose, glucose, sucrose and maltose in food products by high performance liquid chromatography coupled to charged aerosol detector. Microchem J 2014;117:77-82.

133. Lee GO, Kosek P, Lima AA, Singh R, Yori PP, Olortegui MP, et al. Lactulose: mannitol diagnostic test by HPLC and LC-MSMS platforms: considerations for field studies of intestinal barrier function and environmental enteropathy. J Pediatr Gastroenterol Nutr 2014;59:544-50. 
134. Mechri B, Tekaya M, Cheheb $\mathrm{H}$, Hammami M. Determination of mannitol sorbitol and myo-inositol in olive tree roots and rhizospheric soil by gas chromatography and effect of severe drought conditions on their profiles. J Chromatogr Sci 2015;53:1631-8.

135. Butler 0, Forder J, Saunders J. Analytical protocol for the sensitive determination of mannitol, sorbitol and glucose containing powders in pharmaceutical workplaces by ion chromatography using a pulsed amperometric detector. J Pharm Biomed Anal 2015;106:204-9.

136. Dong Y, Yan K, Ma Y, Yang Z, Zhao J, Ding J. A modified LCMS/MS method to simultaneously quantify glycerol and mannitol concentrations in human urine for doping control purposes. J Chromatogr B Anal Technol Biomed Life Sci 2016;1022:153-8.

137. Gervasoni J, Schiattarella A, Giorgio V, Primiano A, Russo C, Tesori V, et al. Validation of an LC-MS/MS method for urinary lactulose and mannitol quantification: results in patients with irritable bowel syndrome. Dis Markers 2016;2016:5340386.

138. Hadjikinova R, Petkova N, Hadjikinov D, Denev P, Hrusavov D. Development and validation of HPLC-RID method for determination of sugars and polyols. J Pharm Sci Res 2017;9:1263-9.

139. Gervasoni J, Primiano A, Graziani C, Scaldaferri F, Gasbarrini A, Urbani A, et al. Validation of UPLC-MS/MS method for determination of urinary lactulose/mannitol. Molecules 2018;23:2705.

140. Musa MA, Kabir M, Hossain MI, Ahmed E, Siddique A, Rashid H, et al. Measurement of intestinal permeability using lactulose and mannitol with conventional five hours and shortened two hours urine collection by two different methods: HPAE-PAD and LC-MSMS. PLoS One 2019;14:e0220397.

141. Noorani B, Chowdhury EA, Alqahtani F, Ahn Y, Patel D, AlAhmad $\mathrm{A}$, et al. LC-MS/MS-based in vitro and in vivo investigation of blood-brain barrier integrity by simultaneous quantitation of mannitol and sucrose. Fluids Barriers CNS 2020;17:61.

142. Wong $\mathrm{Y}$, Or M, Lau H, Wong S, Fung W. Analysis of underivatized alditols in traditional chinese medicines by liquid chromatography-tandem mass spectrometry based on ligand exchange chromatography. Sep Sci Plus 2021;4:6-15.

143. British Pharmacopoeia; London Stationary Office; 2005.

144. USP 27/NF 27; United States Pharmacopoeial Convection, Inc, Rockville; 2004.

145. Indian Pharmacopoeia; Indian Pharmacopoeia Commission, Ghaziabad In; 1996.

146. Farhadi A, Keshavarzian A, Holmes EW, Fields J, Zhang L, Banan A. Gas chromatographic method for detection of urinary sucralose: application to the assessment of intestinal permeability. J Chromatogr B Anal Technol Biomed Life Sci 2003;784:145-54.

147. Marsilio R, D'Antiga L, Zancan L, Dussini N, Zacchello F. Simultaneous HPLC determination with light-scattering detection of lactulose and mannitol in studies of intestinal permeability in pediatrics. Clin Chem 1998;44:1685-91.

148. Delahunty T, Hollander D. Liquid-chromatographic method for estimating urinary sugars: applicability to studies of intestinal permeability. Clin Chem 1986;32:1542-4.

149. Campbell Roberts SN, Williams AC, Grimsey IM, Booth SW. Quantitative analysis of mannitol polymorphs. X-ray powder diffractometry--exploring preferred orientation effects. J Pharm Biomed Anal 2002;28:1149-59.

150. Chen G, Zhang L, Wu X, Ye J. Determination of mannitol and three sugars in ligustrum lucidum ait. by capillary electrophoresis with electrochemical detection. Anal Chim Acta 2005;530:15-21.

151. Paroni R, Fermo I, Molteni L, Folini L, Pastore MR. Lactulose and mannitol intestinal permeability detected by capillary electrophoresis. J Chromatogr B Anal Technol Biomed Life Sci 2006;834:183-7.

152. Pospisilova M, Polasek M, Safra J, Petriska I. Determination of mannitol and sorbitol in infusion solutions by capillary zone electrophoresis using on-column complexation with borate and indirect spectrophotometric detection. J Chromatogr A 2007;1143:258-63.

153. Braun DE, Maas SG, Zencirci N, Langes C, Urbanetz NA, Griesser UJ. Simultaneous quantitative analysis of ternary mixtures of Dmannitol polymorphs by FT-raman spectroscopy and multivariate calibration models. Int J Pharm 2010;385:29-36.

154. Stryeck S, Horvath A, Leber B, Stadlbauer V, Madl T. NMR spectroscopy enables simultaneous quantification of carbohydrates for diagnosis of intestinal and gastric permeability. Sci Rep 2018;8:14650.

155. Cordero C, Cagliero C, Liberto E, Sgorbini B, Rubiolo P, Bicchi C. Gas chromatography. Chem Anal Food 2020. p. 457.

156. AI Ruiz Matute, S Rodriguez-Sa Nchez, ML Sanz, AC Soria. Chromatographic technique: gas chromatography (GC). Modern Techniques Food Authentication; 2018. p. 424.

157. Fang X, Xie J, Chu S, Jiang Y, An Y, Li C, et al. Quadrupole-linear ion trap tandem mass spectrometry system for clinical biomarker analysis. $\quad 2021$. https://doi.org/10.1016/j.eng.2020.10.021.

158. Pereira VR, Hosker BS. Low-cost $(<€ 5)$, open-source, potential alternative to commercial spectrophotometers. PLoS Biol 2019;17:e3000321.

159. Moldoveanu SC, David V. The practice of HPLC, Editor(s): Moldoveanu SC, David V. Selection of the HPLC Method in Chemical Analysis; 2017. p. 464.

160. LaCourse ME, LaCourse WR. General instrumentation in HPLC. Liquid Chromatography; 2017. p. 422.

161. Sun Z, Wang X, Andersson R. Role of intestinal permeability in monitoring mucosal barrier function. History, methodology, and significance of pathophysiology. Dig Surg 1998;1:386-97.

162. Camilleri M, Nadeau A, Lamsam J, Nord SL, Ryks M, Burton D, et al. Understanding measurements of intestinal permeability in healthy humans with urine lactulose and mannitol excretion. J Neurogastroenterol Motil 2010;2:e15-e26.

163. Denno DM, VanBuskirk K, Nelson ZC, Musser CA, Hay Burgess DC, Tarr PI. Use of the lactulose to mannitol ratio to evaluate childhood environmental enteric dysfunction: a systematic review. Clin Infect Dis 2014;59(Suppl 4):S213-S219.

164. Płotka Wasylka J, Gałuszka A, Namiesnik J. Green analytical chemistry: summary of existing knowledge and future trends. J Płotka Wasylka J Namiesnik. (eds.) Green Analytical Chemistry, Green Chemistry and Sustainable Technology, Springer Nature Singapore Pte Ltd; 2019.

165. Gałuszka A, Migaszewski Z, Namiesnik J. The 12 principles of green analytical chemistry and the significance mnemonic of green analytical practices. Trends Anal Chem 2013;50:78-84. 